Supporting Information for

\title{
Rhodium-Catalyzed Intramolecular Conjugate Addition of Vinylstannanes to 2,3- Dihydro-4-pyridones. An Efficient Route to Stereoselective Construction of Indolizidines
}

\author{
Magdalena Dziedzic, Marta Małecka and Bartłomiej Furman* \\ Institute of Organic Chemistry, Polish Academy of Sciences, 01-224 Warsaw, Poland \\ furbar@icho.edu.pl
}

General. Column chromatography was performed on silica gel, Merck grade 60 (230-400 mesh). TLC plates were visualized with UV and staining with phosphomolybdic acid. ${ }^{1} \mathrm{H}$ NMR spectra were recorded on a Bruker AM500 $(500 \mathrm{MHz})$ spectrometer and the chemical shifts are reported in ppm with TMS as an internal standard $(\delta=0 \mathrm{ppm}) .{ }^{13} \mathrm{C}$ NMR spectra were recorded at $125 \mathrm{MHz}$ on a Bruker AM500 spectrometer. The chemical shifts are reported in ppm relative to the center line of the triplet at $77.0 \mathrm{ppm}$ for $\mathrm{CDCl}_{3}$. Infrared (IR) spectra were measured with a Perkin Elmer FT-IR-1600 infrared spectrophotometer. High resolution mass spectra were taken on a Mariner PerSeptive Biosystems mass spectrometer with time-of-flight (TOF) detector. Unless stated otherwise, all reagents and solvents were purchased from commercial sources and used without additional purification. The following known compounds were prepared by literature procedures: (Z)-3-(trimethylstannyl)allylamine, ${ }^{1} 1$-methoxy-3-trimethylsilyloxy-1,3-butadiene, ${ }^{2}\left[\mathrm{Rh}(\mathrm{cod})(\mathrm{MeCN})_{2}\right] \mathrm{BF}_{4} .^{3}$

General Procedure for the Synthesis of Imines with $(Z)$-3-(Trimethylstannyl)-allylamine To a solution of (Z)-3-(trimethylstannyl)-allylamine ( $1 \mathrm{mmol}$ ) in $10 \mathrm{~mL}$ of $\mathrm{CH}_{2} \mathrm{Cl}_{2}$ was added $1 \mathrm{mmol}$ of the corresponding aldehyde and $200 \mathrm{mg}$ of activated $4 \AA$ molecular sieves. The mixture was stirred for $12 \mathrm{~h}$ at ambient temperature and filtered through a Celite pad and the solvent was evaporated in vacuo. The resulting imines were directly used in the subsequent reactions without further purification.

General Procedure for the aza-Diels-Alder Reaction of Danishefsky's Diene with Imines To a solution of $1.0 \mathrm{mmol}$ of the respective imine in $\mathrm{MeCN}(10 \mathrm{~mL})$ was added $\mathrm{Yb}(\mathrm{OTf})_{3}$ $(0.1 \mathrm{mmol})$ followed by 1.2 equiv of the diene $4(1.2 \mathrm{mmol})$. The reaction mixture was stirred for 6-12 $\mathrm{h}$ at room temperature. After addition of saturated $\mathrm{NaHCO}_{3}(10 \mathrm{~mL})$, the aqueous phase was extracted with $\mathrm{CH}_{2} \mathrm{Cl}_{2}(10 \mathrm{~mL} \times 3)$, the combined organic phases were dried with $\mathrm{MgSO}_{4}$, and the solvent was evaporated in vacuo. The crude material was purified by flash chromatography on silica gel with $\mathrm{Et}_{2} \mathrm{O}$-hexane mixtures as eluents.<smiles>C[AsH2]/C=C\CN1C=CC(=O)CC1c1ccccc1</smiles>

Analytical data for 2-phenyl-1-(3-trimethylstannanyl-allyl)-2,3-dihydro-1H-pyridin-4one: Chromatography $\left(80 \% \mathrm{Et}_{2} \mathrm{O} /\right.$ hexane) afforded $0.327 \mathrm{~g}(87 \%)$ of a yellow oil; $500-\mathrm{MHz}$ ${ }^{1} \mathrm{H}$ NMR $\left(\mathrm{CDCl}_{3}\right) \delta$ 7.35-7.23 (m, 5H), $7.16(\mathrm{~d}, \mathrm{~J}=7.7 \mathrm{~Hz}, 1 \mathrm{H}), 6.40(\mathrm{dq}, \mathrm{J}=12.8,5.2 \mathrm{~Hz}, 1$ $\mathrm{H}), 6.13(\mathrm{~d}, \mathrm{~J}=12.8 \mathrm{~Hz}, 1 \mathrm{H}), 5.03(\mathrm{~d}, \mathrm{~J}=7.7 \mathrm{~Hz}, 1 \mathrm{H}), 4.58(\mathrm{~m}, 1 \mathrm{H}), 3.71$ (ddd, J = 14.8, 5.2, 1.6 Hz, 1H), 3.55 (ddd, J = 14.8, 7.9, $0.9 \mathrm{~Hz}, 1 \mathrm{H}), 2.86$ (dd, J = 16.4, 7.0 Hz, 1H), 2.64 $(\mathrm{dd}, \mathrm{J}=16.4,7.8 \mathrm{~Hz}, 1 \mathrm{H}), 0.01(\mathrm{~s}, 9 \mathrm{H}) ; 125-\mathrm{MHz}^{13} \mathrm{C}$ NMR $\left(\mathrm{CDCl}_{3}\right) \delta 190.2,153.1,142.1$, 138.7, 136.1, 129.0, 128.2, 126.8, 99.0, 61.4, 57.3, 43.9, -8.7; IR (neat) $\mathrm{cm}^{-1} 1642,1591$; Anal. calcd for $\mathrm{C}_{17} \mathrm{H}_{23} \mathrm{NOSn}$ : C, 54.29; H, 6.16; N, 3.72. Found: C, 54.46; H, 6.10; N, 3.50. 
<smiles>CS/C=C\CN1C=CC(=O)CC1c1ccc(C)cc1</smiles>

Analytical data for 2-p-tolyl-1-(3-trimethylstannanyl-allyl)-2,3-dihydro-1 $H$-pyridin-4-one: Chromatography $\left(80 \% \mathrm{Et}_{2} \mathrm{O} /\right.$ hexane) afforded $0.359 \mathrm{~g}(92 \%)$ of a yellow oil; $500-\mathrm{MHz}{ }^{1} \mathrm{H}$ $\operatorname{NMR}\left(\mathrm{CDCl}_{3}\right) \delta$ 7.19-7.14 (m, 4H), $7.16(\mathrm{~d}, \mathrm{~J}=7.7 \mathrm{~Hz}, 1 \mathrm{H}), 6.41(\mathrm{dq}, \mathrm{J}=12.8,5.2 \mathrm{~Hz}, 1 \mathrm{H})$, $6.14(\mathrm{~d}, \mathrm{~J}=12.8 \mathrm{~Hz}, 1 \mathrm{H}), 5.05$ (d, J = 7.7 Hz, $1 \mathrm{H}$ ), 4.57 (dd, J = 8.6, $6.8 \mathrm{~Hz}, 1 \mathrm{H}$ ), 3.70 (ddd, $\mathrm{J}=14.8,5.2,1.6 \mathrm{~Hz}, 1 \mathrm{H}), 3.55$ (ddd, $\mathrm{J}=14.8,7.7,1.1 \mathrm{~Hz}, 1 \mathrm{H}), 2.83$ (dd, $\mathrm{J}=16.4,6.8 \mathrm{~Hz}$, $1 \mathrm{H}), 2.67(\mathrm{dd}, \mathrm{J}=16.4,8.6 \mathrm{~Hz}, 1 \mathrm{H}), 2.34(\mathrm{~s}, 3 \mathrm{H}), 0.05(\mathrm{~s}, 9 \mathrm{H}) ; 125-\mathrm{MHz}{ }^{13} \mathrm{C} \mathrm{NMR}\left(\mathrm{CDCl}_{3}\right) \delta$ 190.5, 153.2, 142.3, 138.1, 136.0, 135.7, 129.7, 126.8, 99.1, 61.5, 57.2, 44.2, 21.1, -8.7; IR (neat) $\mathrm{cm}^{-1} 1645,1591$; HRMS calcd. for $\mathrm{C}_{18} \mathrm{H}_{25} \mathrm{NOSn} 391.09581$, found 391.09414.<smiles>COc1ccc(C2CC(=O)C=CN2C/C=C\SC)cc1</smiles>

Analytical data for 2-(4-methoxy-phenyl)-1-(3-trimethylstannanyl-allyl)-2,3-dihydro-1Hpyridin-4-one: Chromatography $\left(70 \% \mathrm{Et}_{2} \mathrm{O} /\right.$ hexane) afforded $0.345 \mathrm{~g}(85 \%)$ of a yellow oil; 500-MHz ${ }^{1} \mathrm{H}$ NMR $\left(\mathrm{CDCl}_{3}\right) \delta 7.21(\mathrm{~d}, 2 \mathrm{H}), 7.15(\mathrm{~d}, \mathrm{~J}=7.7 \mathrm{~Hz}, 1 \mathrm{H}), 6.88(\mathrm{~d}, 2 \mathrm{H}), 6.41(\mathrm{dq}, \mathrm{J}$ $=12.8,5.2 \mathrm{~Hz}, 1 \mathrm{H}), 6.15(\mathrm{~d}, \mathrm{~J}=12.8 \mathrm{~Hz}, 1 \mathrm{H}), 5.06(\mathrm{~d}, \mathrm{~J}=7.7 \mathrm{~Hz}, 1 \mathrm{H}), 4.56(\mathrm{dd}, \mathrm{J}=8.3$, $6.9 \mathrm{~Hz}, 1 \mathrm{H}), 3.80(\mathrm{~s}, 3 \mathrm{H}), 3.69$ (ddd, J = 14.9, 5.3, 1.5 Hz, 1H), 3.55 (dd, J = 14.9, $7.7 \mathrm{~Hz}$, $1 \mathrm{H}), 2.82(\mathrm{dd}, \mathrm{J}=16.4,6.9 \mathrm{~Hz}, 1 \mathrm{H}), 2.67(\mathrm{dd}, \mathrm{J}=16.4,8.4 \mathrm{~Hz}, 1 \mathrm{H}), 0.06(\mathrm{~s}, 9 \mathrm{H}) ; 125-\mathrm{MHz}$ ${ }^{13} \mathrm{C} \mathrm{NMR}\left(\mathrm{CDCl}_{3}\right) \delta 190.6,159.6,153.1,142.3,135.9,130.8,128.1,114.5,99.0,61.2,57.1$, 55.3, 44.2, -8.7; IR (neat) $\mathrm{cm}^{-1} 1647,1576$; HRMS calcd. for $\mathrm{C}_{18} \mathrm{H}_{25} \mathrm{NO}_{2} \mathrm{Sn} 407.0907$, found 407.0888 .<smiles>C[AsH2]/C=C\CN1C=CC(=O)CC1c1ccc(Cl)cc1</smiles>

Analytical data for 2-(4-chloro-phenyl)-1-(3-trimethylstannanyl-allyl)-2,3-dihydro-1Hpyridin-4-one: Chromatography $\left(80 \% \mathrm{Et}_{2} \mathrm{O} /\right.$ hexane) afforded $0.369 \mathrm{~g}(90 \%)$ of a yellow oil; $500-\mathrm{MHz}^{1} \mathrm{H}$ NMR $\left(\mathrm{CDCl}_{3}\right) \delta 7.31(\mathrm{~m}, 2 \mathrm{H}), 7.21(\mathrm{~m}, 2 \mathrm{H}), 7.16(\mathrm{~d}, \mathrm{~J}=7.7 \mathrm{~Hz}, 1 \mathrm{H}), 6.40(\mathrm{dq}$, $\mathrm{J}=12.8,5.2 \mathrm{~Hz}, 1 \mathrm{H}), 6.16(\mathrm{~d}, \mathrm{~J}=12.8 \mathrm{~Hz}, 1 \mathrm{H}), 5.05(\mathrm{~d}, \mathrm{~J}=7.7 \mathrm{~Hz}, 1 \mathrm{H}), 4.58(\mathrm{t}, \mathrm{J}=7.2 \mathrm{~Hz}$, $1 \mathrm{H}), 3.73(\mathrm{ddd}, \mathrm{J}=14.8,5.2,1.5 \mathrm{~Hz}, 1 \mathrm{H}), 3.55(\mathrm{ddd}, \mathrm{J}=14.8,7.8,0.9 \mathrm{~Hz}, 1 \mathrm{H}), 2.88(\mathrm{dd}, \mathrm{J}=$ 16.4, 7.1 Hz, 1H), $2.59(\mathrm{dd}, \mathrm{J}=16.4,7.3 \mathrm{~Hz}, 1 \mathrm{H}), 0.04(\mathrm{~s}, 9 \mathrm{H}) ; 125-\mathrm{MHz}{ }^{13} \mathrm{C} \mathrm{NMR}\left(\mathrm{CDCl}_{3}\right)$ $\delta 189.7,152.9,141.9,137.2,136.5,134.1,129.3,128.2,99.3,60.7,57.5,43.7,-8.7$; IR (neat) $\mathrm{cm}^{-1} 1654,1566$; HRMS calcd. for $\mathrm{C}_{17} \mathrm{H}_{22} \mathrm{NONaClSn}(\mathrm{M}+\mathrm{Na})$ 434.0304, found 434.0288. 
<smiles>C[As]/C=C\CN1C=CC(=O)CC1c1ccco1</smiles>

Analytical data for 2-furan-2-yl-1-(3-trimethylstannanyl-allyl)-2,3-dihydro-1 $\mathrm{H}$-pyridin-4one: Chromatography $\left(80 \% \mathrm{Et}_{2} \mathrm{O} /\right.$ hexane) afforded $0.285 \mathrm{~g}(78 \%)$ of a yellow oil; $500-\mathrm{MHz}$ ${ }^{1} \mathrm{H} \mathrm{NMR}\left(\mathrm{CDCl}_{3}\right) \delta 7.40(\mathrm{~d}, \mathrm{~J}=2.0 \mathrm{~Hz}, 1 \mathrm{H}), 7.01(\mathrm{~d}, \mathrm{~J}=7.7 \mathrm{~Hz}, 1 \mathrm{H}), 6.47(\mathrm{dq}, \mathrm{J}=12.9,5.8$ $\mathrm{Hz}, 1 \mathrm{H}), 6.35(\mathrm{dd}, \mathrm{J}=2.0,3.3 \mathrm{~Hz}, 1 \mathrm{H}), 6.30(\mathrm{~d}, \mathrm{~J}=3.3 \mathrm{~Hz}, 1 \mathrm{H}), 6.23(\mathrm{~d}, \mathrm{~J}=12.9 \mathrm{~Hz}, 1 \mathrm{H})$, $5.03(\mathrm{~d}, \mathrm{~J}=7.7 \mathrm{~Hz}, 1 \mathrm{H}), 4.68(\mathrm{dd}, \mathrm{J}=6.8,5.1 \mathrm{~Hz}, 1 \mathrm{H}), 3.91-3.81(\mathrm{~m}, 2 \mathrm{H}), 2.89(\mathrm{dd}, \mathrm{J}=16.4$, $6.9 \mathrm{~Hz}, 1 \mathrm{H}), 2.76(\mathrm{dd}, \mathrm{J}=16.4,5.1 \mathrm{~Hz}, 1 \mathrm{H}), 0.15(\mathrm{~s}, 9 \mathrm{H}) ; 125-\mathrm{MHz}{ }^{13} \mathrm{C} \mathrm{NMR}\left(\mathrm{CDCl}_{3}\right) \delta$ $190.2,151.6,151.2,142.5,142.2,136.6,110.4,108.5,98.5,58.3,54.3,39.9$, -8.6; IR (neat) $\mathrm{cm}^{-1} 1641,1582$; HRMS calcd. for $\mathrm{C}_{15} \mathrm{H}_{22} \mathrm{NO}_{2} \mathrm{Sn}(\mathrm{M}+\mathrm{H}) 368.0667$, found 368.0681 .<smiles>C[As]/C=C\CN1C=CC(=O)CC1COCc1ccccc1</smiles>

Analytical data for 2-benzyloxymethyl-1-(3-trimethylstannanyl-allyl)-2,3-dihydro-1Hpyridin-4-one: Chromatography $\left(70 \% \mathrm{Et}_{2} \mathrm{O} /\right.$ hexane) afforded $0.374 \mathrm{~g}(89 \%)$ of a yellow oil; $500-\mathrm{MHz}{ }^{1} \mathrm{H} \mathrm{NMR}\left(\mathrm{CDCl}_{3}\right) \delta$ 7.36-7.25 $(\mathrm{m}, 5 \mathrm{H}), 6.97(\mathrm{dd}, \mathrm{J}=7.5,1.1 \mathrm{~Hz}, 1 \mathrm{H}), 6.44(\mathrm{dq}, \mathrm{J}=$ 12.9, $5.9 \mathrm{~Hz}, 1 \mathrm{H}), 6.16(\mathrm{~d}, \mathrm{~J}=12.9 \mathrm{~Hz}, 1 \mathrm{H}), 4.89(\mathrm{dd}, \mathrm{J}=7.5,0.9 \mathrm{~Hz}, 1 \mathrm{H}), 4.49(\mathrm{~s}, 2 \mathrm{H})$, 3.97-3.85 (m, $2 \mathrm{H}), 3.83$ (dd, J = 9.4, $7.9 \mathrm{~Hz}, 1 \mathrm{H}), 3.78-3.72(\mathrm{~m}, 1 \mathrm{H}), 3.46(\mathrm{dd}, \mathrm{J}=9.4,5.1$ $\mathrm{Hz}, 1 \mathrm{H}), 2.77$ (dd, J = 16.7, 7.3 Hz, 1H), 2.36 (ddd, J = 16.7, 2.5, 1.0 Hz, 1 H), 0.18 (s, 9H); $125-\mathrm{MHz}{ }^{13} \mathrm{C} \mathrm{NMR}\left(\mathrm{CDCl}_{3}\right) \delta 190.1,151.4,142.9,137.6,136.2,128.5,127.8,127.8,98.1$, 73.6, 68.5, 59.5, 56.5, 37.7, -8.6; IR (neat) $\mathrm{cm}^{-1} 1642$, 1586; HRMS calcd. for $\mathrm{C}_{19} \mathrm{H}_{27} \mathrm{NO}_{2} \mathrm{Sn}$ 421.10638 , found 421.10461 .<smiles>C[AsH2]/C=C\CN1C=CC(=O)CC1C(C)C</smiles>

Analytical data for 2-isopropyl-1-(3-trimethylstannanyl-allyl)-2,3-dihydro-1 $\mathrm{H}$-pyridin-4one: Chromatography $\left(60 \% \mathrm{Et}_{2} \mathrm{O} /\right.$ hexane) afforded $0.236 \mathrm{~g}(69 \%)$ of a yellow oil; $500-\mathrm{MHz}$ ${ }^{1} \mathrm{H} \mathrm{NMR}\left(\mathrm{CDCl}_{3}\right) \delta 7.01(\mathrm{~d}, \mathrm{~J}=7.4 \mathrm{~Hz}, 1 \mathrm{H}), 6.45(\mathrm{dq}, \mathrm{J}=12.8,5.4 \mathrm{~Hz}, 1 \mathrm{H}), 6.19(\mathrm{~d}, \mathrm{~J}=$ $12.8 \mathrm{~Hz}, 1 \mathrm{H}$ ), 4.89 (d, J = 7.4 Hz, $1 \mathrm{H}), 3.87$ (ddd, J = 15.0, 5.4, $1.5 \mathrm{~Hz}, 1 \mathrm{H}$ ), 3.79 (ddd, J = 15.0, 7.6, 1.0 Hz, $1 \mathrm{H}), 3.26(\mathrm{~m}, 1 \mathrm{H}), 2.67$ (dd, J = 16.6, 7.6 Hz, 1H), 2.43 (dd, J = 16.6, 3.9 $\mathrm{Hz}, 1 \mathrm{H}), 2.22(\mathrm{~m}, 1 \mathrm{H}), 0.95(\mathrm{~d}, \mathrm{~J}=6.8 \mathrm{~Hz}, 6 \mathrm{H}) 0.25(\mathrm{~s}, 9 \mathrm{H}) ; 125-\mathrm{MHz}{ }^{13} \mathrm{C} \mathrm{NMR}\left(\mathrm{CDCl}_{3}\right) \delta$ 191.1, 152.3, 142.9, 135.7, 97.6, 62.0, 59.0, 36.3, 29.0, 19.5, 17.7, -8.6; IR (neat) cm ${ }^{-1} 1640$, 1585; HRMS calcd. for $\mathrm{C}_{14} \mathrm{H}_{26} \mathrm{NOSn}(\mathrm{M}+\mathrm{H}) 344.1031$, found 344.1045. 
<smiles>CO/C=C\CN1C=CC(=O)CC1C1CCCCC1</smiles>

Analytical data for 2-cycloheksyl-1-(3-trimethylstannanyl-allyl)-2,3-dihydro-1 $H$-pyridin4-one: Chromatography (50\% $\mathrm{Et}_{2} \mathrm{O} /$ hexane) afforded $0.275 \mathrm{~g}(72 \%)$ of a yellow oil; 500 $\mathrm{MHz}{ }^{1} \mathrm{H} \mathrm{NMR}\left(\mathrm{CDCl}_{3}\right) \delta 7.00(\mathrm{~d}, \mathrm{~J}=7.4 \mathrm{~Hz}, 1 \mathrm{H}), 6.46(\mathrm{dq}, \mathrm{J}=12.6,5.5 \mathrm{~Hz}, 1 \mathrm{H}), 6.19(\mathrm{~d}, \mathrm{~J}$ $=12.6 \mathrm{~Hz}, 1 \mathrm{H}), 4.86(\mathrm{~d}, \mathrm{~J}=7.4 \mathrm{~Hz}, 1 \mathrm{H}), 3.86(\mathrm{ddd}, \mathrm{J}=14.9,5.5,1.5 \mathrm{~Hz}, 1 \mathrm{H}), 3.78$ (ddd, J $=15.0,8.6,1.0 \mathrm{~Hz}, 1 \mathrm{H}), 3.25(\mathrm{~m}, 1 \mathrm{H}), 2.70(\mathrm{dd}, \mathrm{J}=16.6,7.8 \mathrm{~Hz}, 1 \mathrm{H}), 2.44(\mathrm{dd}, \mathrm{J}=16.6,2.0$ $\mathrm{Hz}, 1 \mathrm{H}), 1.85(\mathrm{~m}, 1 \mathrm{H}), 1.80-1.54(\mathrm{~m}, 6 \mathrm{H}), 1.25-1.10(\mathrm{~m}, 4 \mathrm{H}) \quad 0.25(\mathrm{~s}, 9 \mathrm{H}) ; 125-\mathrm{MHz}{ }^{13} \mathrm{C}$ $\operatorname{NMR}\left(\mathrm{CDCl}_{3}\right) \delta 191.0,152.2,142.9,135.7,97.4,61.5,59.3,39.4,37.0,29.8,28.5,26.3$, 26.1, -8.6; IR (neat) $\mathrm{cm}^{-1} 1641,1586$; HRMS calcd. for $\mathrm{C}_{17} \mathrm{H}_{30} \mathrm{NOSn}(\mathrm{M}+\mathrm{H})$ 384.13442, found 384.13354 .

General Procedure for the Catalytic Intramolecular Conjugate Addition of Vinylstannanes to 2,3-Dihydro-4-pyridones. To a solution $0.5 \mathrm{mmol}$ of the respective 2,3dihydro-4-pyridone in 1,4-dioxane $(4 \mathrm{~mL})$ was added $[\mathrm{RhCl}(\operatorname{cod})]_{2}(0.025 \mathrm{mmol})$. After stirring for $2-3 \mathrm{~h}$ at room temperature the reaction mixture was concentrated. The crude material was purified by flash chromatography on silica gel with $\mathrm{Et}_{2} \mathrm{O}$-hexane mixtures as eluents.<smiles>O=C1C[C@H]2C=CCN2[C@H](c2ccccc2)C1</smiles>

Analytical data for 5-phenyl-5,6,8,8a-tetrahydro-3H-indolizin-7-one: Chromatography $\left(30 \% \mathrm{Et}_{2} \mathrm{O} /\right.$ hexane) afforded $0.089 \mathrm{~g}(84 \%)$ of a brown oil; $500-\mathrm{MHz}{ }^{1} \mathrm{H} \mathrm{NMR}\left(\mathrm{CDCl}_{3}\right) \delta$ 7.41-7.25 (m, 5 H), 5.86 (dq, J = 6.2, 1.9 Hz, 1 H), 5.80 (dq, J = 6.2, 1.9 Hz, 1 H), 4.57 (m, 1 $\mathrm{H}), 4.15(\mathrm{dd}, \mathrm{J}=12.3,2.9 \mathrm{~Hz}, 1 \mathrm{H}), 3.77(\mathrm{dq}, \mathrm{J}=14.4,1.9 \mathrm{~Hz}, 1 \mathrm{H}), 3.41$ (dddd, $\mathrm{J}=14.4,8.5$, 4.4, $2.0 \mathrm{~Hz}, 1 \mathrm{H}), 2.75(\mathrm{~m}, 1 \mathrm{H}), 2.61-2.48(\mathrm{~m}, 3 \mathrm{H}) ; 125-\mathrm{MHz}{ }^{13} \mathrm{C} \mathrm{NMR}\left(\mathrm{CDCl}_{3}\right) \delta 209.8$, $131.1,130.1,128.6,127.6,127.5,126.9,62.6,62.5,60.0,48.7,43.6$; IR (neat) $\mathrm{cm}^{-1} 2928$, 1718; HRMS calcd. for $\mathrm{C}_{14} \mathrm{H}_{15} \mathrm{NO} 213.11536$, found 213.11513 .<smiles>Cc1ccc([C@@H]2CC(=O)C[C@H]3C=CCN32)cc1</smiles>

Analytical data for 5-p-tolyl-5,6,8,8a-tetrahydro-3H-indolizin-7-one: Chromatography (30\% $\mathrm{Et}_{2} \mathrm{O} /$ hexane) afforded $0.097 \mathrm{~g}(86 \%)$ of a brown oil; $500-\mathrm{MHz}{ }^{\mathrm{l}} \mathrm{H} \mathrm{NMR}\left(\mathrm{CDCl}_{3}\right) \delta$ 7.29-7.14 (m, $4 \mathrm{H}), 5.85$ (dq, J = 6.2, $1.9 \mathrm{~Hz}, 1 \mathrm{H}), 5.79$ (dq, J = 6.2, 2.1 Hz, $1 \mathrm{H}), 4.55$ (m, 1 H), 4.12 (dd, J = 12.2, 2.9 Hz, 1 H), 3.77 (dq, J = 14.5, 1.9 Hz, $1 \mathrm{H}$ ), 3.41 (dddd, J = 14.5, 8.6, 
4.3, $2.1 \mathrm{~Hz}, 1 \mathrm{H}), 2.72(\mathrm{dd}, \mathrm{J}=17.4,12.2 \mathrm{~Hz} 1 \mathrm{H}), 2.57-2.48(\mathrm{~m}, 3 \mathrm{H}), 2.34(\mathrm{~s}, 3 \mathrm{H}) ; 125-\mathrm{MHz}$ ${ }^{13} \mathrm{C} \mathrm{NMR}\left(\mathrm{CDCl}_{3}\right) \delta 210.0,140.1,137.1,130.1,129.3,127.7,126.6,62.4,62.3,60.0,48.8$, 43.7, 21.1; IR (neat) $\mathrm{cm}^{-1}$ 2920, 1716; HRMS calcd. for $\mathrm{C}_{15} \mathrm{H}_{17} \mathrm{NO}$ 227.13101, found 213.13156 .<smiles>COc1ccc([C@@H]2CC(=O)C[C@H]3C=CCN32)cc1</smiles>

Analytical data for 5-(4-methoxy-phenyl)-5,6,8,8a-tetrahydro-3H-indolizin-7-one: Chromatography $\left(30 \% \mathrm{Et}_{2} \mathrm{O} /\right.$ hexane) afforded $0.099 \mathrm{~g}(82 \%)$ of a brown oil; $500-\mathrm{MHz}{ }^{1} \mathrm{H}$ NMR $\left(\mathrm{CDCl}_{3}\right) \delta$ 7.32-6.86 (m, $\left.4 \mathrm{H}\right), 5.86(\mathrm{~m}, 1 \mathrm{H}), 5.79(\mathrm{~m}, 1 \mathrm{H}), 4.53(\mathrm{~m}, 1 \mathrm{H}), 4.11(\mathrm{dd}, \mathrm{J}=$ 12.1, $2.8 \mathrm{~Hz}, 1 \mathrm{H}), 3.81(\mathrm{~s}, 3 \mathrm{H}), 3.75(\mathrm{dq}, \mathrm{J}=14.5,2.0 \mathrm{~Hz}, 1 \mathrm{H}), 3.41$ (dddd, J = 14.5, 8.4, 4.2, $2.0 \mathrm{~Hz}, 1 \mathrm{H}), 2.71(\mathrm{dd}, \mathrm{J}=17.3,12.2 \mathrm{~Hz} 1 \mathrm{H}), 2.58-2.46(\mathrm{~m}, 3 \mathrm{H}) ; 125-\mathrm{MHz}{ }^{13} \mathrm{C} \mathrm{NMR}$ $\left(\mathrm{CDCl}_{3}\right) \delta 210.1,158.9,135.1,130.1,127.9,127.6,114.1$ 62.4, 61.9, 60.0, 55.4, 48.8, 43.6; IR (neat) $\mathrm{cm}^{-1}$ 2943, 1717; HRMS calcd. for $\mathrm{C}_{15} \mathrm{H}_{17} \mathrm{NO}_{2} 243.12593$, found 243.12608.<smiles>O=C1C[C@H]2C=CCN2[C@H](c2ccc(Cl)cc2)C1</smiles>

Analytical data for 5-(4-chloro-phenyl)-5,6,8,8a-tetrahydro-3H-indolizin-7-one: Chromatography $\left(30 \% \mathrm{Et}_{2} \mathrm{O} /\right.$ hexane) afforded $0.097 \mathrm{~g}(79 \%)$ of a brown oil; $500-\mathrm{MHz}{ }^{1} \mathrm{H}$ NMR $\left(\mathrm{CDCl}_{3}\right) \delta$ 7.36-7.32 (m, $\left.4 \mathrm{H}\right), 5.86(\mathrm{dq}, \mathrm{J}=5.9,1.9 \mathrm{~Hz}, 1 \mathrm{H}), 5.79(\mathrm{~m}, 1 \mathrm{H}), 4.55(\mathrm{~m}, 1$ H), 4.13 (dd, J = 12.3, $2.9 \mathrm{~Hz}, 1 \mathrm{H}), 3.76(\mathrm{dq}, \mathrm{J}=14.3,2.0 \mathrm{~Hz}, 1 \mathrm{H}), 3.37$ (dddd, $\mathrm{J}=14.3,8.6$, 4.3, $2.0 \mathrm{~Hz}, 1 \mathrm{H}), 2.68(\mathrm{dd}, \mathrm{J}=17.3,12.3 \mathrm{~Hz} 1 \mathrm{H}), 2.56-2.42(\mathrm{~m}, 3 \mathrm{H}) ; 125-\mathrm{MHz}{ }^{13} \mathrm{C} \mathrm{NMR}$ $\left(\mathrm{CDCl}_{3}\right) \delta 209.3,141.6,133.1,130.1,128.8,128.2,127.6,62.5,62.1,60.0,48.6,43.6$; IR (neat) $\mathrm{cm}^{-1}$ 1716; HRMS calcd. for $\mathrm{C}_{14} \mathrm{H}_{15} \mathrm{CINO}(\mathrm{M}+\mathrm{H}) 248.0837$, found 248.0849.<smiles>O=C1CC(c2ccco2)N2CC=C[C@H]2C1</smiles>

Analytical data for 5-furan-2-yl-5,6,8,8a-tetrahydro-3H-indolizin-7-one: Chromatography $\left(50 \% \mathrm{Et}_{2} \mathrm{O} /\right.$ hexane) afforded $0.087 \mathrm{~g}(86 \%)$ of a brown oil; $400-\mathrm{MHz}{ }^{1} \mathrm{H} \mathrm{NMR}\left(\mathrm{CDCl}_{3}\right) \delta$ $7.38(\mathrm{dd}, \mathrm{J}=1.8,0.8 \mathrm{~Hz}, 1 \mathrm{H}), 6.32(\mathrm{dd}, \mathrm{J}=3.3,1.8 \mathrm{~Hz}, 1 \mathrm{H}), 6.19(\mathrm{dt}, \mathrm{J}=3.3,0.8 \mathrm{~Hz}, 1 \mathrm{H})$, $5.89(\mathrm{dq}, \mathrm{J}=6.0,2.1 \mathrm{~Hz}, 1 \mathrm{H}), 5.78(\mathrm{~m}, 1 \mathrm{H}), 4.40(\mathrm{dd}, \mathrm{J}=7.6,5.1 \mathrm{~Hz}, 1 \mathrm{H}), 4.21(\mathrm{~m}, 1 \mathrm{H})$, $3.85(\mathrm{~m}, 1 \mathrm{H}), 3.67(\mathrm{~m}, 1 \mathrm{H}), 2.85-2.72(\mathrm{~m}, 2 \mathrm{H}), 2.55(\mathrm{ddd}, \mathrm{J}=15.5,3.3,0.8 \mathrm{~Hz}, 1 \mathrm{H}), 2.44$ $(\mathrm{dd}, \mathrm{J}=15.5,11.9 \mathrm{~Hz}, 1 \mathrm{H}) ; 100-\mathrm{MHz}^{13} \mathrm{C} \mathrm{NMR}\left(\mathrm{CDCl}_{3}\right) \delta 208.6,154.4,142.2,130.7,127.9$, $110.0,107.1,61.6,58.1,54.6,44.8,44.2$; IR (neat) $\mathrm{cm}^{-1} 2922$, 1716; HRMS calcd. for $\mathrm{C}_{12} \mathrm{H}_{13} \mathrm{NO}_{2} 203.09463$, found 203.09443. 


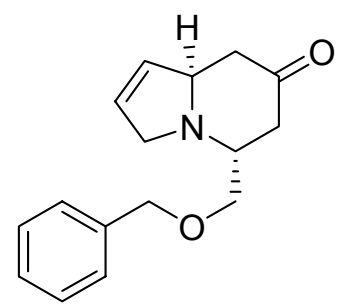

Analytical data for 5-benzyloxymethyl-5,6,8,8a-tetrahydro-3H-indolizin-7-one: Chromatography $\left(30 \% \mathrm{Et}_{2} \mathrm{O} /\right.$ hexane) afforded $0.115 \mathrm{~g}(90 \%)$ of a brown oil; $500-\mathrm{MHz}{ }^{1} \mathrm{H}$ NMR $\left(\mathrm{CDCl}_{3}\right) \delta$ 7.37-7.26 (m, $\left.5 \mathrm{H}\right), 5.87(\mathrm{dq}, \mathrm{J}=6.2,1.9 \mathrm{~Hz}, 1 \mathrm{H}), 5.78(\mathrm{dq}, \mathrm{J}=6.2,2.0 \mathrm{~Hz}$, $1 \mathrm{H}), 4.54(\mathrm{~s}, 2 \mathrm{H}), 4.38(\mathrm{~m}, 1 \mathrm{H}), 4.04(\mathrm{dq}, \mathrm{J}=14.0,2.0 \mathrm{~Hz}, 1 \mathrm{H}), 3.60(\mathrm{~m}, 1 \mathrm{H}), 3.55(\mathrm{dd}, \mathrm{J}=$ 9.4, 4.6 Hz, 1 H), $3.44(\mathrm{dd}, \mathrm{J}=9.4,5.9 \mathrm{~Hz}, 1 \mathrm{H}), 3.34(\mathrm{~m}, 1 \mathrm{H}), 2.62(\mathrm{dd}, \mathrm{J}=16.7,3.8 \mathrm{~Hz}, 1$ H), $2.50(\mathrm{dd}, \mathrm{J}=16.7,10.1 \mathrm{~Hz}, 1 \mathrm{H}), 2.41(\mathrm{dd}, \mathrm{J}=16.1,3.3 \mathrm{~Hz}, 1 \mathrm{H}), 2.31(\mathrm{dd}, \mathrm{J}=16.1$, 12.0); 125-MHz ${ }^{13} \mathrm{C}$ NMR $\left(\mathrm{CDCl}_{3}\right) \delta 209.4,138.0,130.7,128.4,127.6,127.5,127.4,73.4$, 73.1, 62.6, 59,5, 57,8, 43.8, 42.3; IR (neat) $\mathrm{cm}^{-1} 2928$, 1722; HRMS calcd. for $\mathrm{C}_{16} \mathrm{H}_{19} \mathrm{NO}_{2}$ 257.14158 , found 257.14225 .<smiles>CC(C)[C@H]1CC(=O)C[C@H]2C=CCN21</smiles>

Analytical data for 5-isopropyl-5,6,8,8a-tetrahydro-3H-indolizin-7-one: Chromatography $\left(30 \% \mathrm{Et}_{2} \mathrm{O} /\right.$ hexane) afforded $0.072 \mathrm{~g}(80 \%)$ of a brown oil; $500-\mathrm{MHz}{ }^{1} \mathrm{H} \mathrm{NMR}\left(\mathrm{CDCl}_{3}\right) \delta$ $5.88(\mathrm{dq}, \mathrm{J}=6.1,1.8 \mathrm{~Hz}, 1 \mathrm{H}), 5.78(\mathrm{dq}, \mathrm{J}=6.1,1.9 \mathrm{~Hz}, 1 \mathrm{H}), 4.34(\mathrm{~m}, 1 \mathrm{H}), 3.96(\mathrm{dq}, \mathrm{J}=$ 13.9, $1.8 \mathrm{~Hz}, 1 \mathrm{H}), 3.48$ (dddd, J = 13.9, 8.6, 4.3, $2.1 \mathrm{~Hz}, 1 \mathrm{H}), 2.92(\mathrm{~m}, 1 \mathrm{H}), 2.41-2.28$ (m, 4 $\mathrm{H}), 1.84(\mathrm{~m}, 1 \mathrm{H}), 0.90(\mathrm{~d}, \mathrm{~J}=6.9,6 \mathrm{H}) ; 125-\mathrm{MHz}{ }^{13} \mathrm{C} \mathrm{NMR}\left(\mathrm{CDCl}_{3}\right) \delta 211.2,130.8,127.3$, $63.5,62.5,59.5,43.5,39.4,30.5,16.0$; IR (neat) $\mathrm{cm}^{-1}$ 2957, 1721; HRMS calcd. for $\mathrm{C}_{11} \mathrm{H}_{17} \mathrm{NO} 179.13101$, found 179.13041.<smiles>O=C1CC2C=CCN2C(C2CCCCC2)C1</smiles>

Analytical data for 5-cyclohexyl-5,6,8,8a-tetrahydro-3H-indolizin-7-one: Chromatography (30\% $\mathrm{Et}_{2} \mathrm{O} /$ hexane) afforded $0.084 \mathrm{~g}(77 \%)$ of a brown oil; $500-\mathrm{MHz}{ }^{1} \mathrm{H}$ NMR $\left(\mathrm{CDCl}_{3}\right) \delta$ $5.88(\mathrm{dq}, \mathrm{J}=6.2,1.8 \mathrm{~Hz}, 1 \mathrm{H}), 5.78(\mathrm{dq}, \mathrm{J}=6.2,2.0 \mathrm{~Hz}, 1 \mathrm{H}), 4.31(\mathrm{~m}, 1 \mathrm{H}), 3.96(\mathrm{dq}, \mathrm{J}=$ 13.8, $1.8 \mathrm{~Hz}, 1 \mathrm{H}), 3.52(\mathrm{~m}, 1 \mathrm{H}), 2.93(\mathrm{~m}, 1 \mathrm{H}), 2.42-2.40(\mathrm{~m}, 2 \mathrm{H}), 2.36-2.24(\mathrm{~m}, 2 \mathrm{H}), 1.90$ $(\mathrm{m}, 1 \mathrm{H}), 1.77(\mathrm{~m}, 2 \mathrm{H}), 1.67(\mathrm{~m}, 2 \mathrm{H}), 1.44(\mathrm{~m}, 1 \mathrm{H}), 1.28-1.07$ (m, $3 \mathrm{H}), 1.04-0.80(\mathrm{~m}, 2 \mathrm{H})$; $125-\mathrm{MHz}{ }^{13} \mathrm{C}$ NMR $\left(\mathrm{CDCl}_{3}\right) \delta 211.2,131.1,127.4,63.1,62.4,59.3,43.8,41.1,40.5,30.1$, 27.4, 26.8, 26.7, 26.4; IR (neat) $\mathrm{cm}^{-1} 2928,1714$; HRMS calcd. for $\mathrm{C}_{14} \mathrm{H}_{22} \mathrm{NO}(\mathrm{M}+\mathrm{H})$ 220.16567 , found 220.16545 .

\section{References}

1. Corriu, R. J. P.; Geng, B.; Moreau, J. J. E. J. Org. Chem. 1993, 58, 1443.

2. Hansson, L.; Carlson, R. Acta Chem. Scand. 1989, 43, 188.

3. Green, M.; Kuc, T. A.; Taylor, S. H. J. Chem. Soc. A 1971, 2334. 

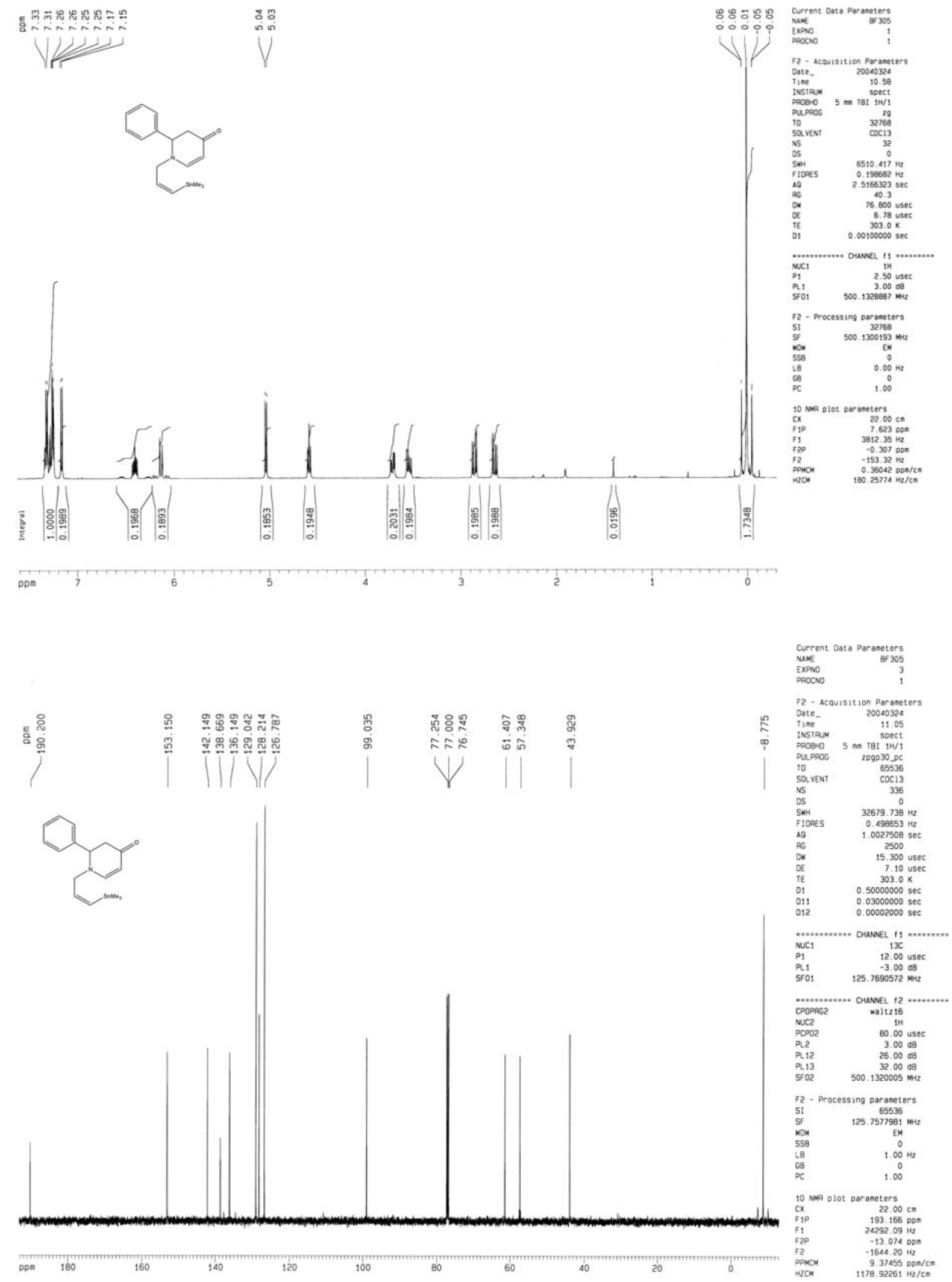

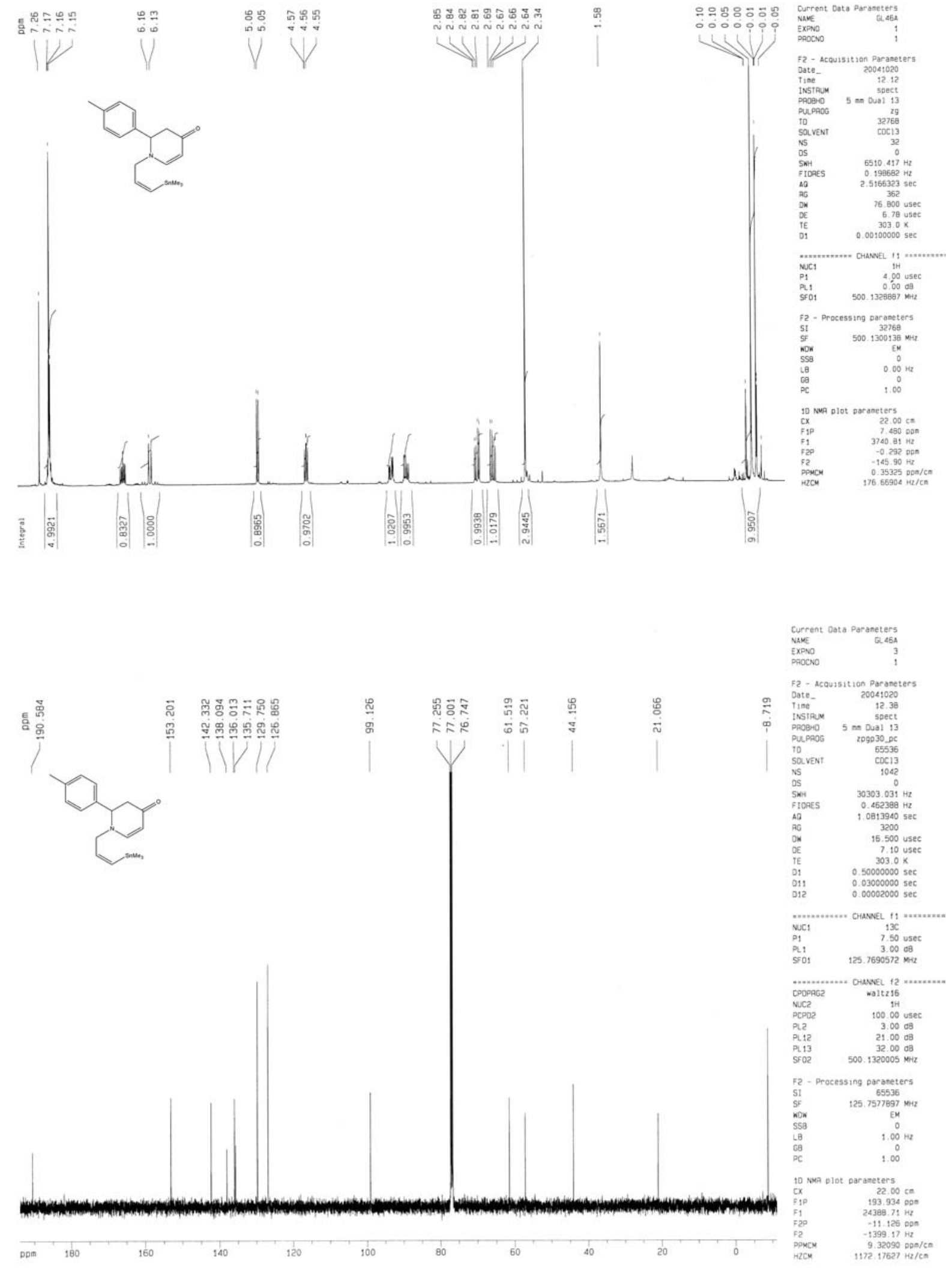

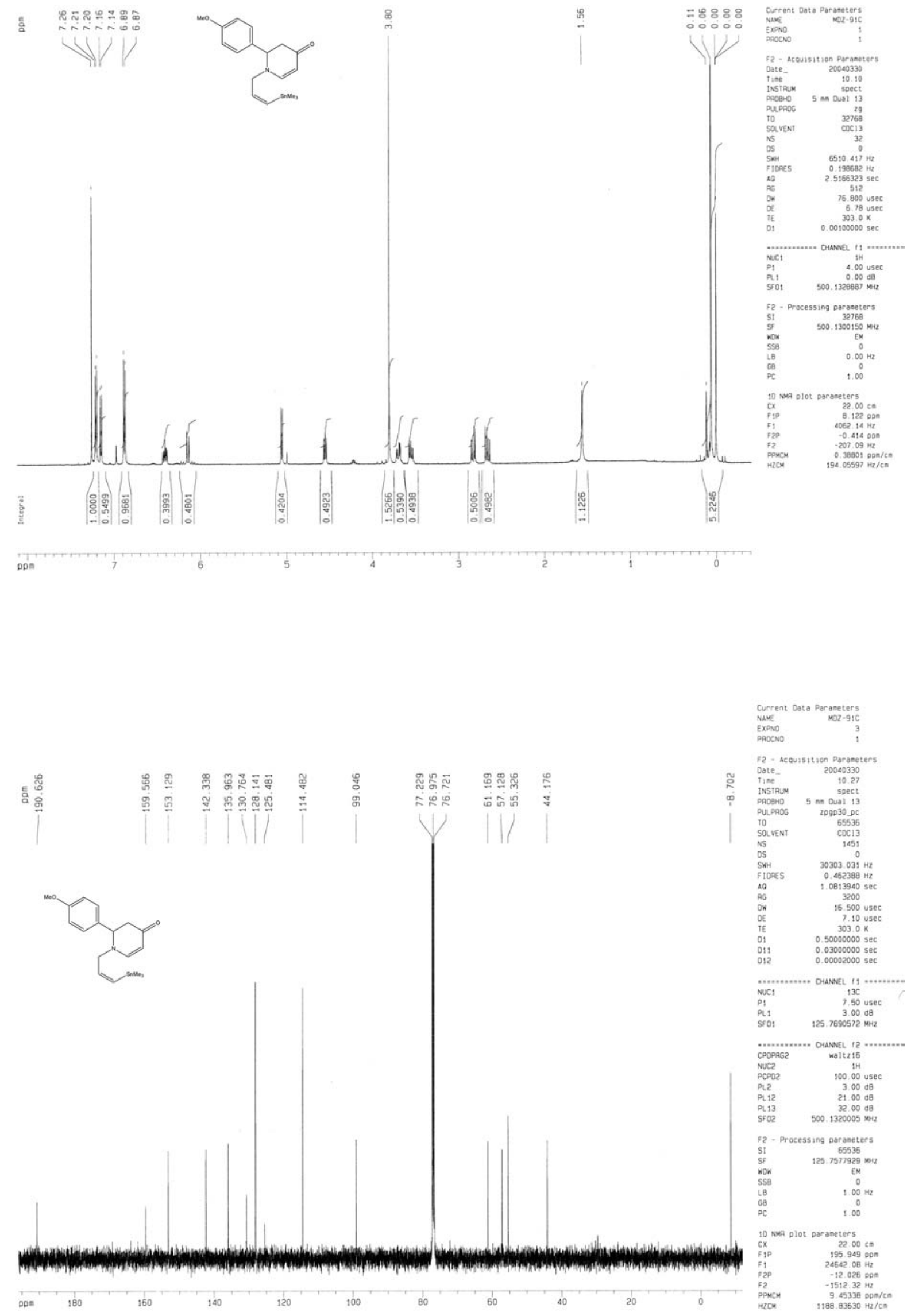

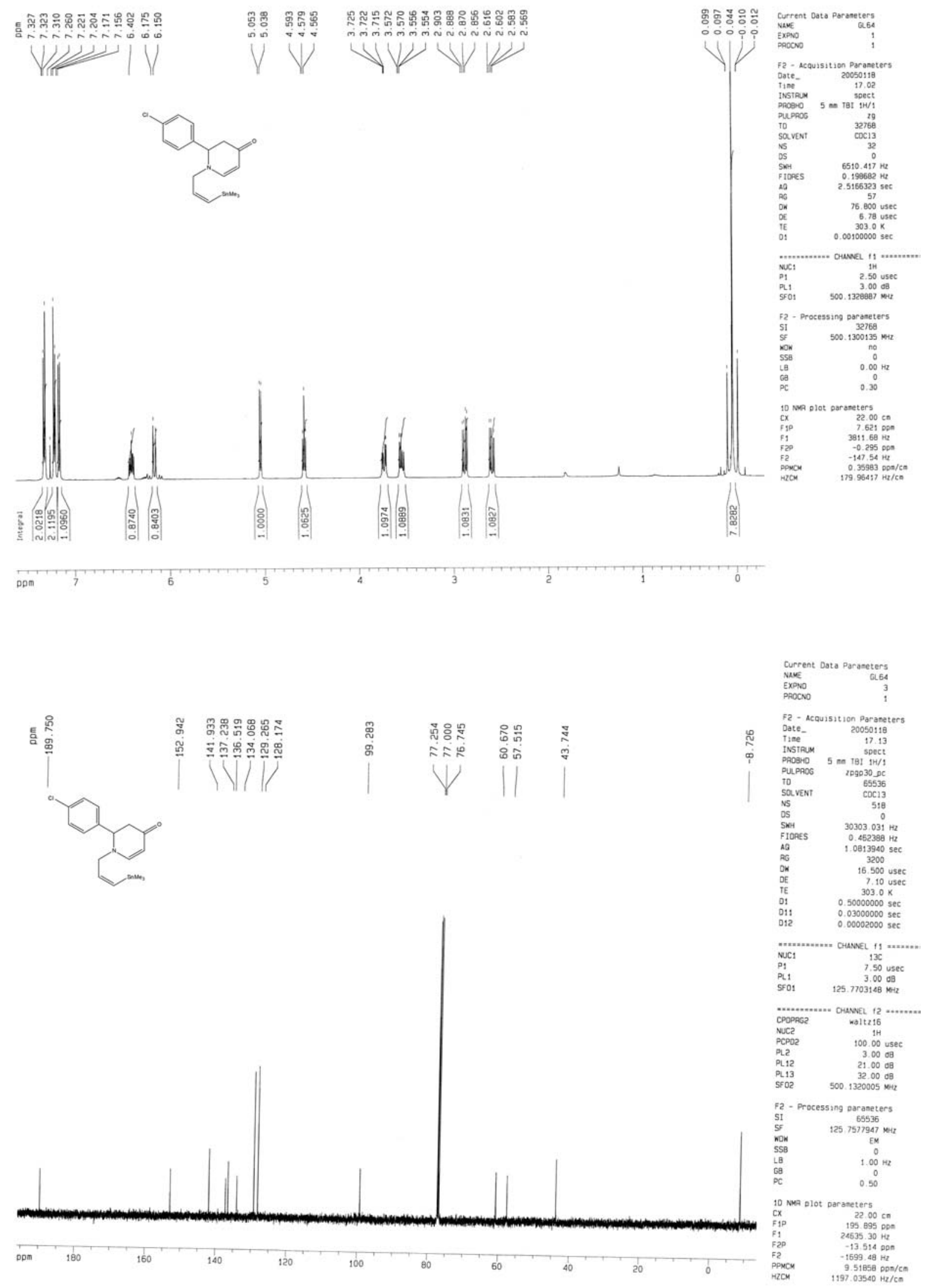

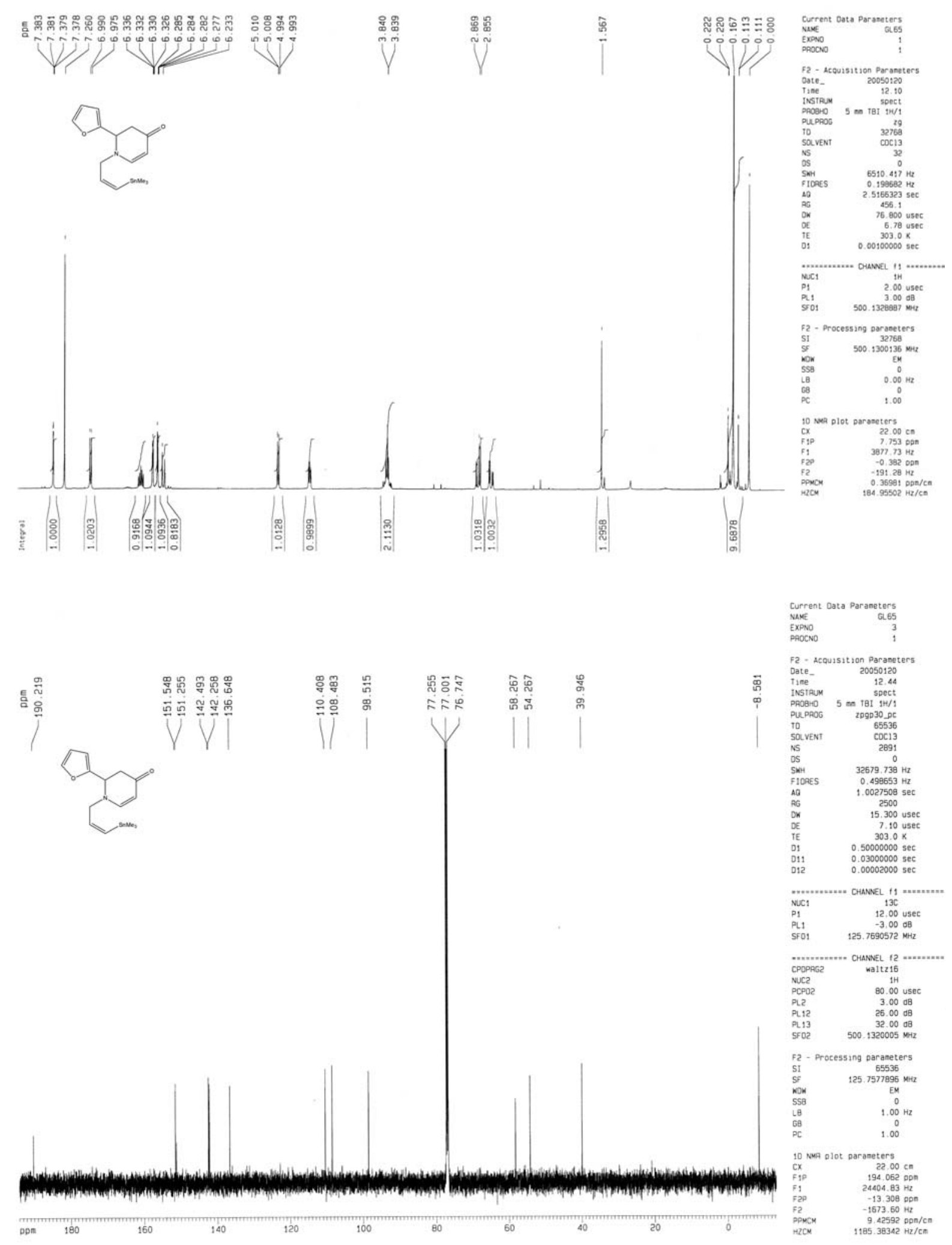

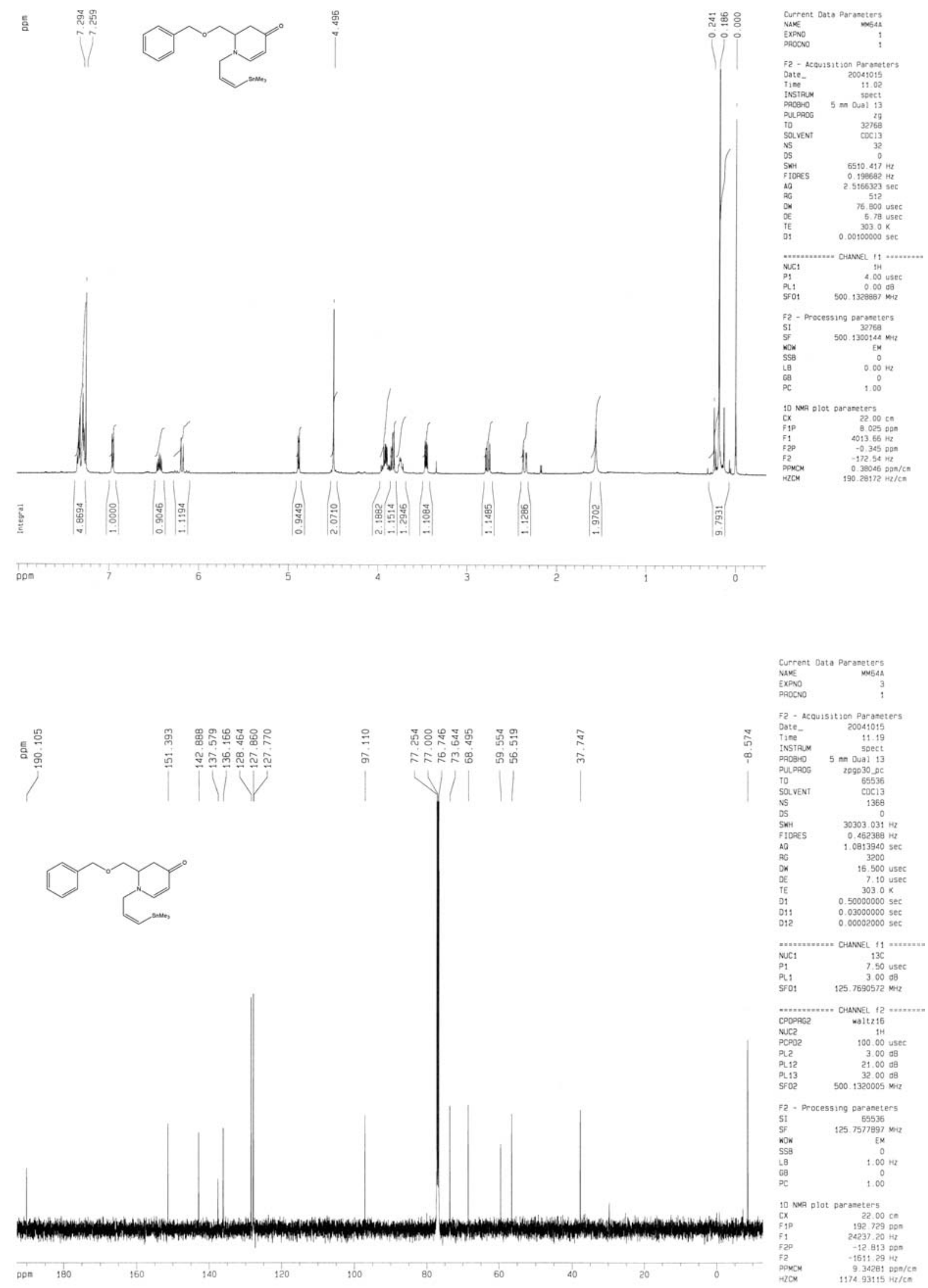

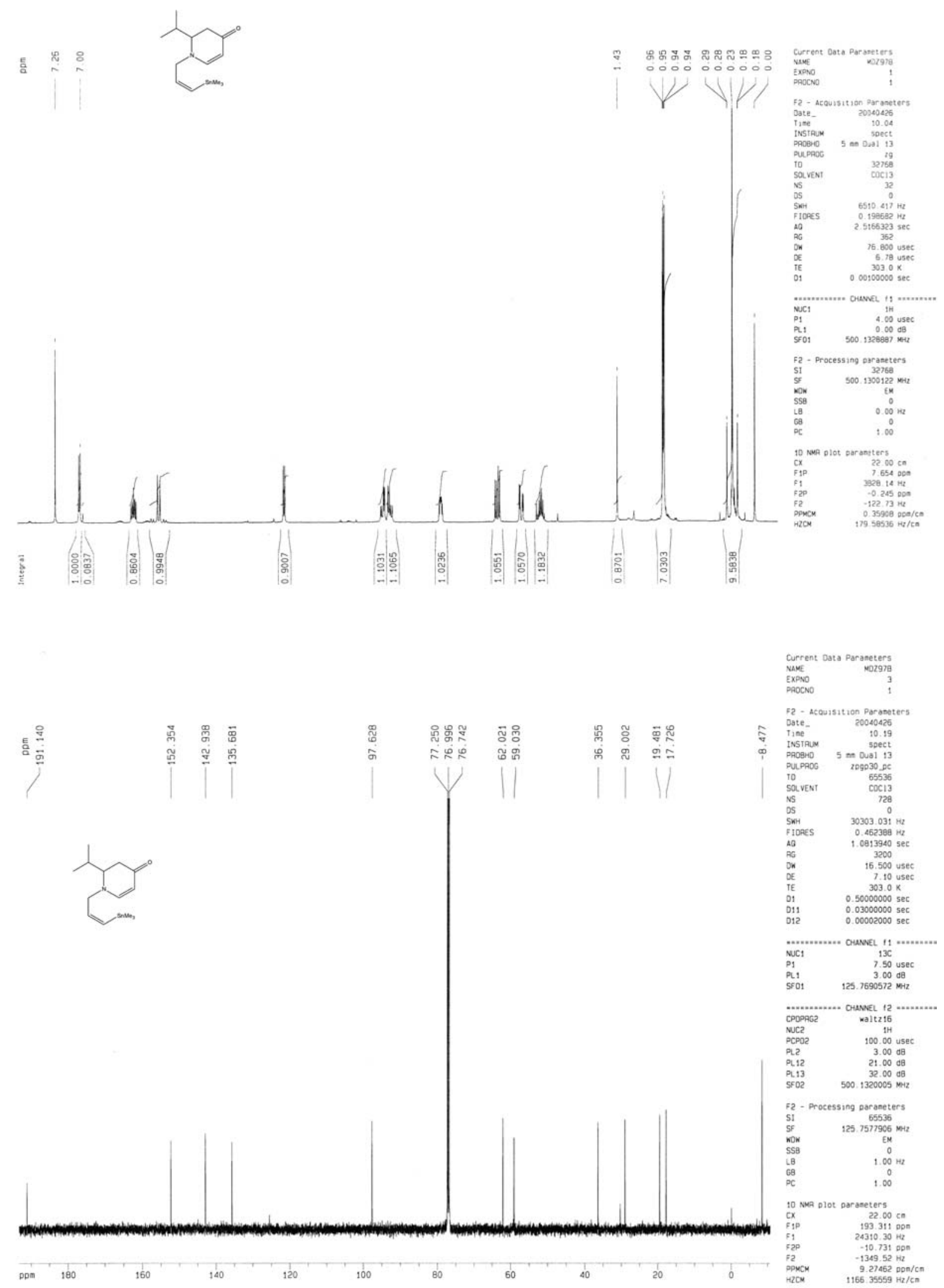

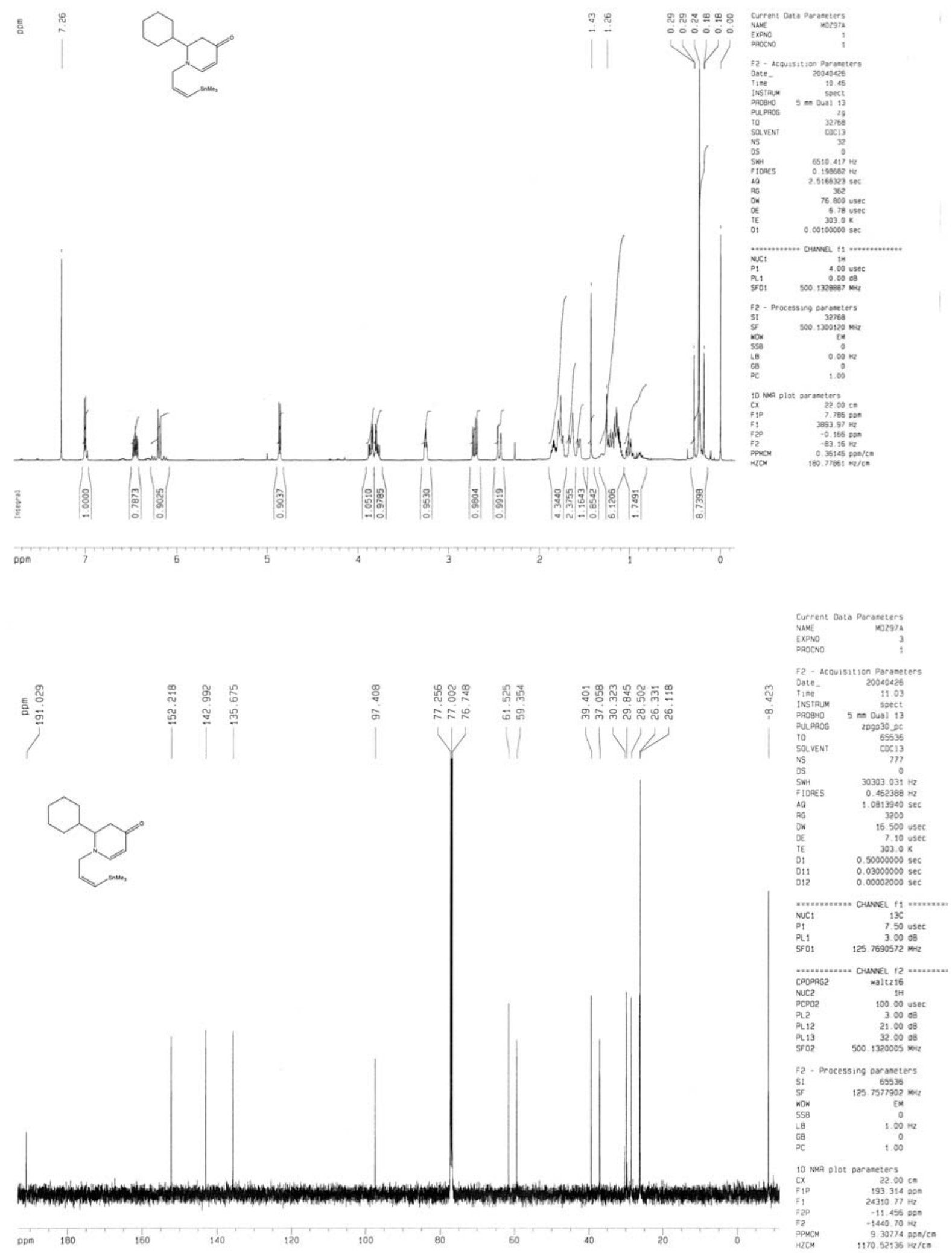

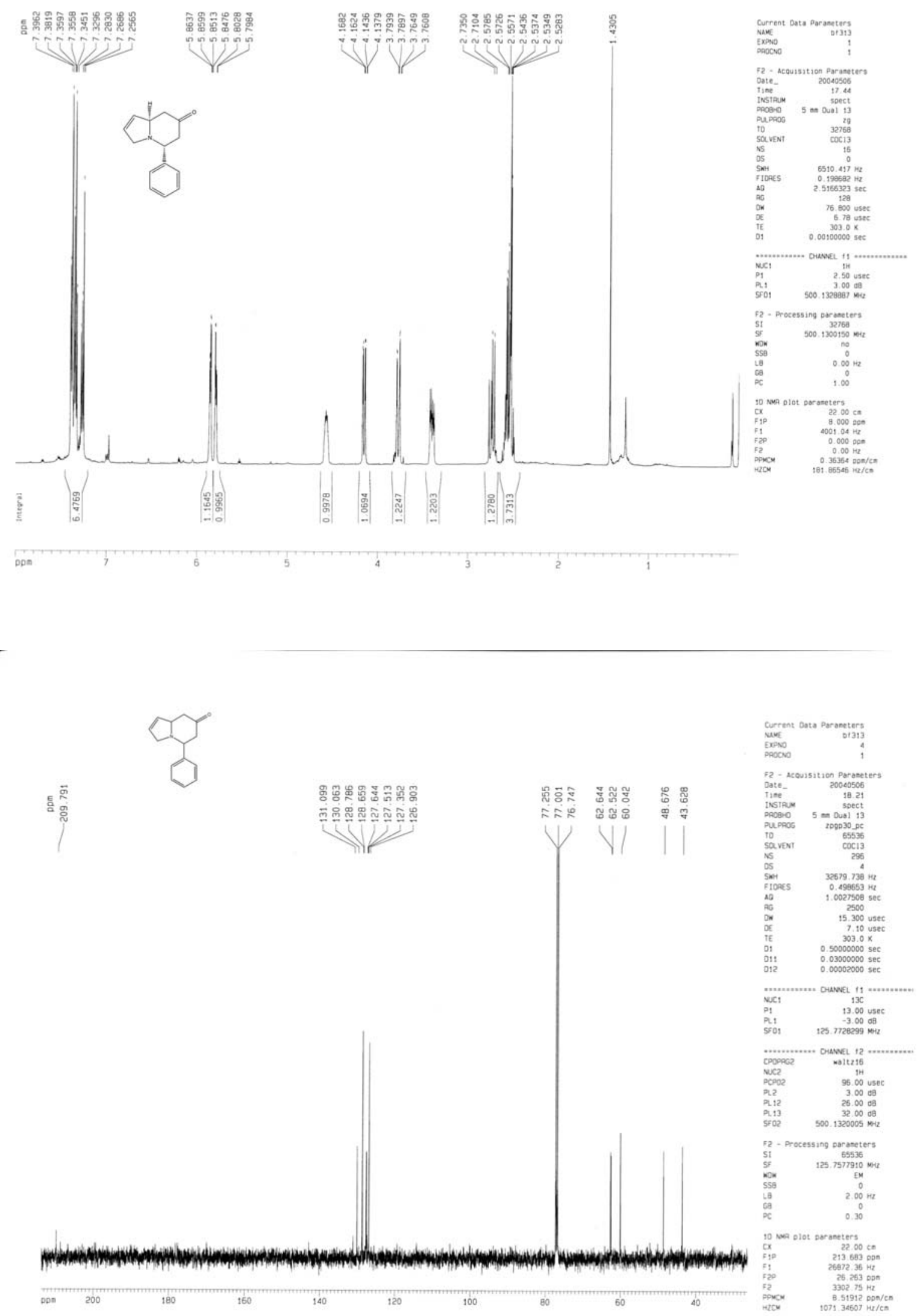

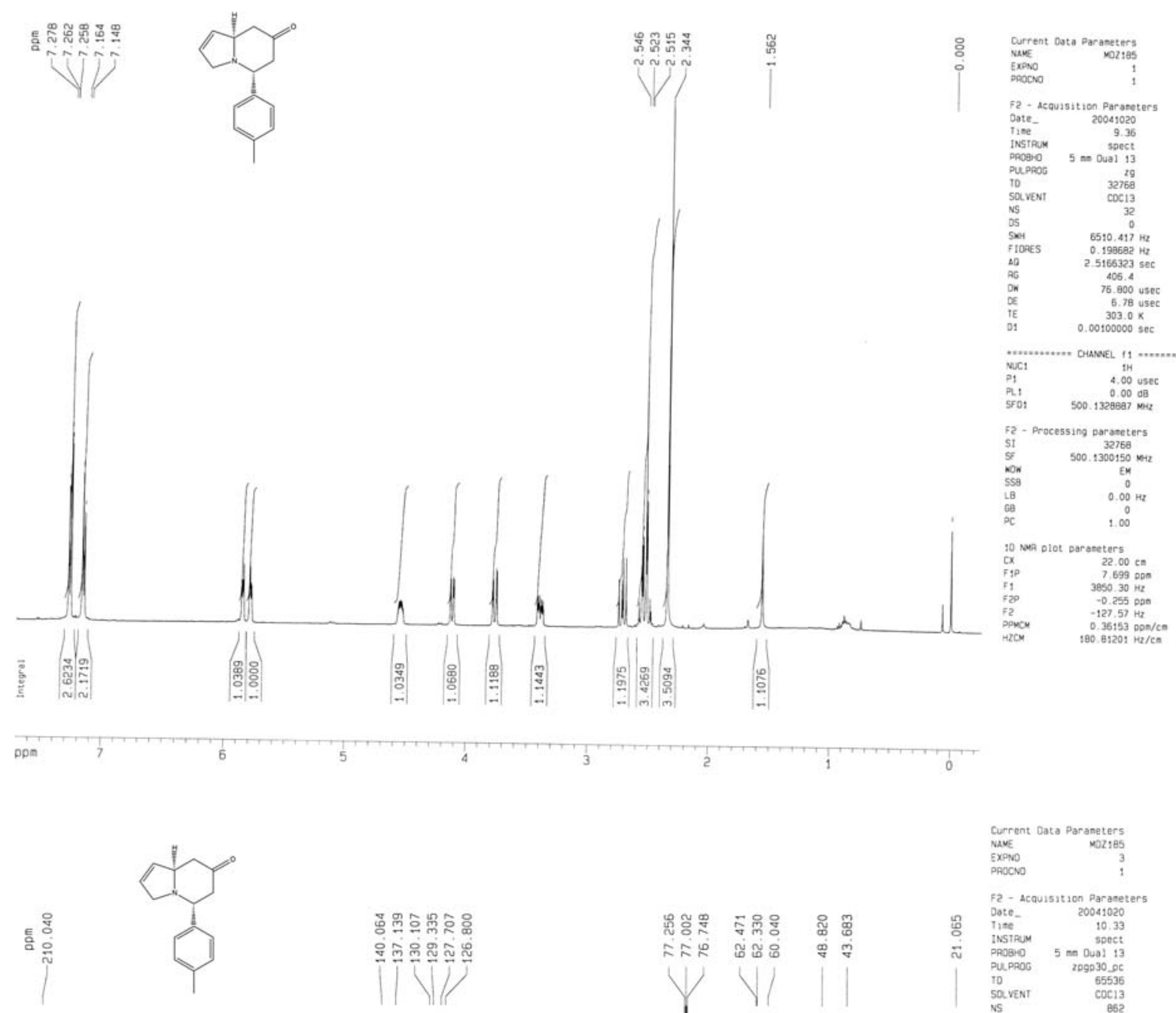

Current Data Paraneters
NavE
EXPNO
EROCONO

2- Acquisition Paraneters

20041020
Date- $\quad 10.33$

InSTRUM spect

(PRDBro $5 \mathrm{~mm}$ Dual 13

$\begin{array}{lr}\text { To } & 65536 \\ \text { Savent } & \text { COC13 } \\ \text { OSt } & \end{array}$

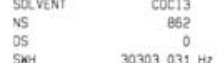

$\begin{array}{ll}\text { Shit } & 30303.031 \mathrm{~Hz} \\ \text { Foees } & 0.462388 \mathrm{~Hz}\end{array}$

$\begin{array}{ll}28 & 1.0613940 \mathrm{sec} \\ 26 & 3200\end{array}$

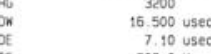

$303.0 \mathrm{~K}$
$0.50000000 \mathrm{sec}$

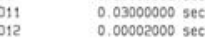

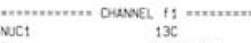

$\begin{array}{ll}13 \mathrm{C} \\ \mathrm{NuCt} & 7.50 \text { uses }\end{array}$

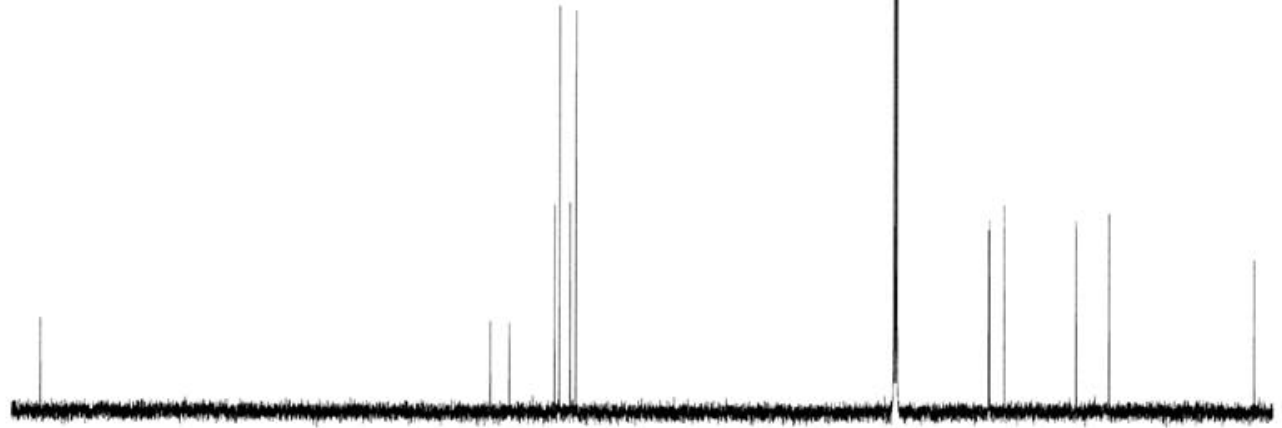

25. $7726299 \mathrm{vin}$

CPDPRG

$\begin{array}{ll}\text { Noper } & \text { Naitzit } \\ \text { IH }\end{array}$

$\begin{array}{ll}100.00 \text { uset } \\ \text { Let } & 3.00 \mathrm{dg}\end{array}$

31.0000
513

G502 500.1320005 Net3

2. - Pracessing paraneters

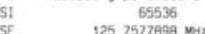

non

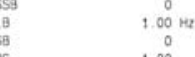

10 NaA giot naraneters
$2200 \mathrm{~cm}$

$\mathrm{Cx}$
$\mathrm{Cl}$
$\mathrm{Fip}$

$18.150 \mathrm{DPm}$
$2282.54 \mathrm{~Hz}$

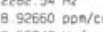

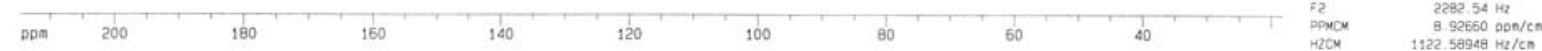



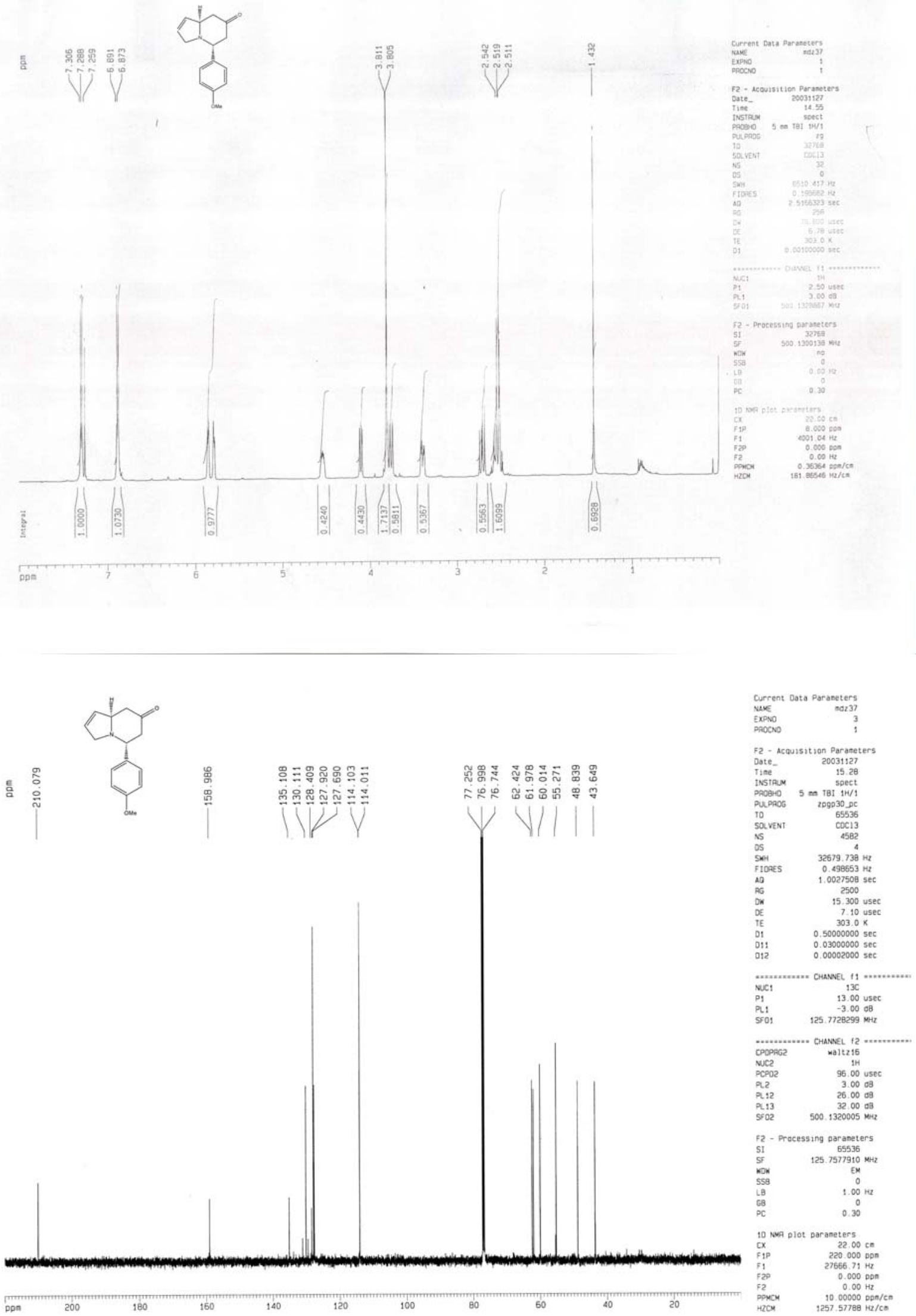

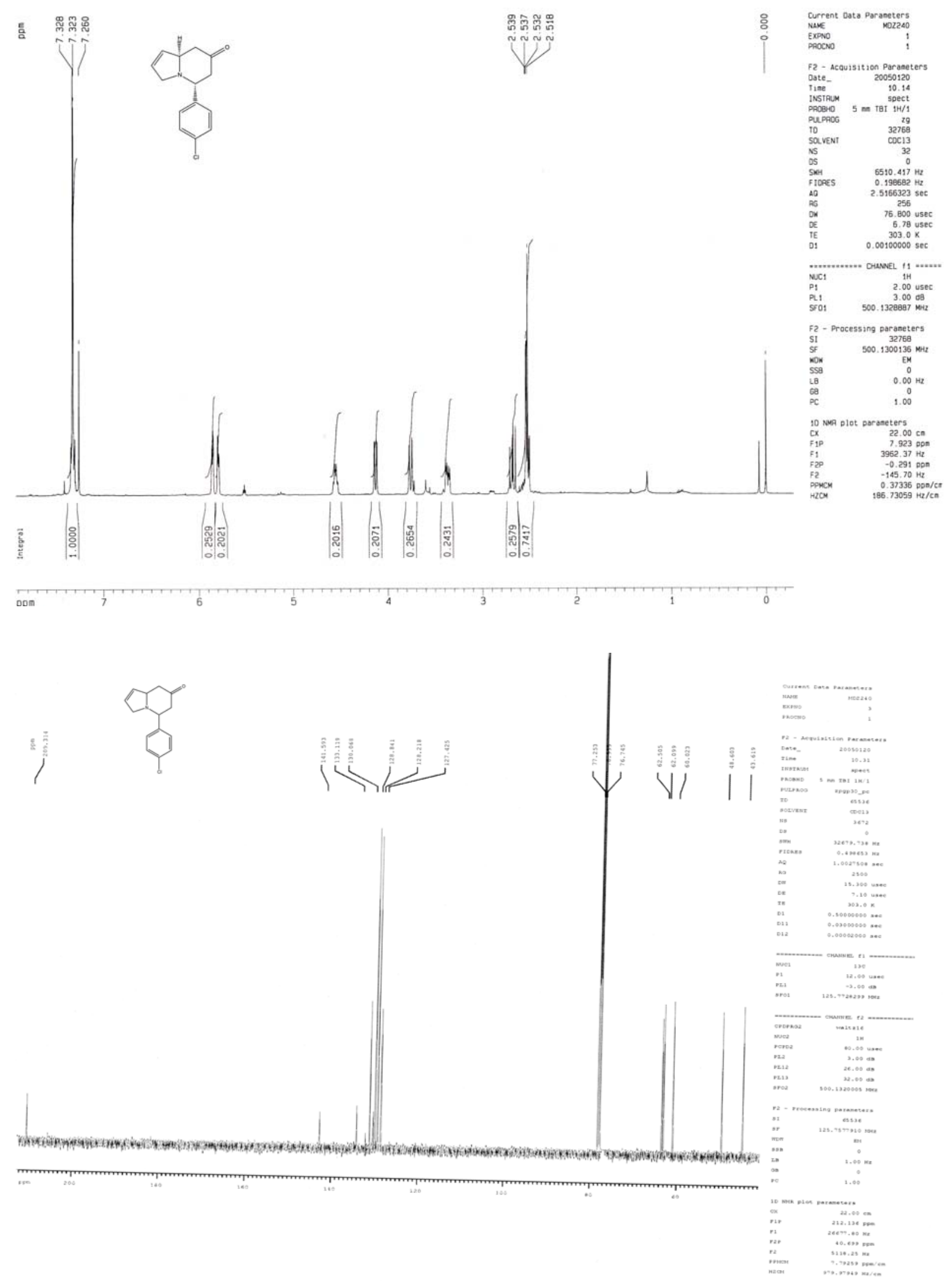
B. Fursan
CHM MOZ 254

Pulse Sequence: s2pul

Solvent: COC13
Ambient temperature

File: chmmdz254 "Schilfsun"
Mercury-400BB "Scho

Relax. de lay $1.000 \mathrm{sec}$

Pulse 33.3 degrees

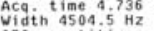

OBSERVE H1 400.0918311 MH

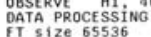
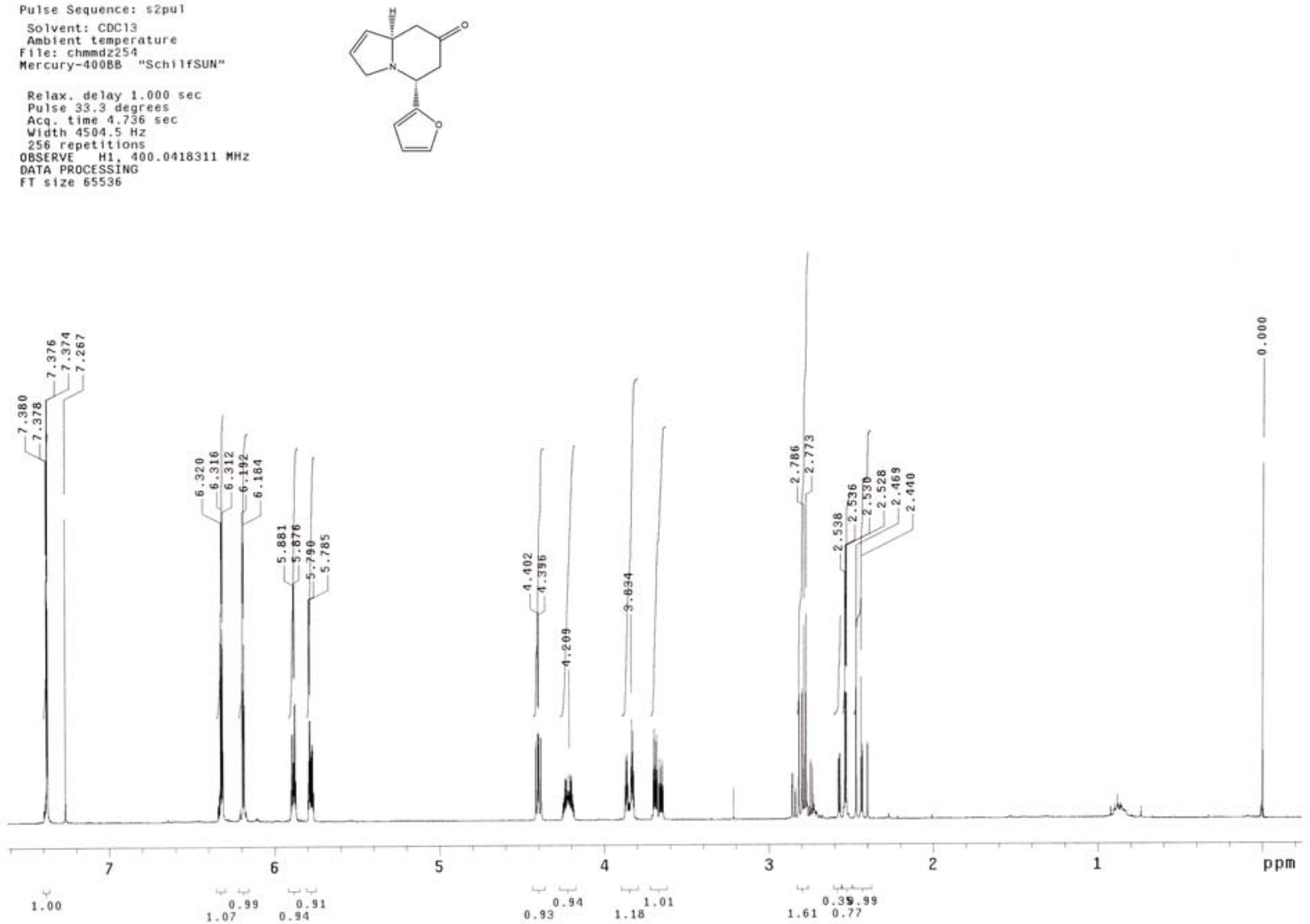

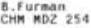

wise Sequence: $52 \mathrm{pu}$

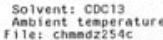

hercury $4000 \mathrm{BE}$ "

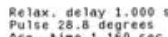

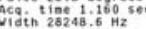

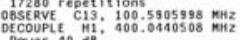

contingusti on

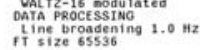

के
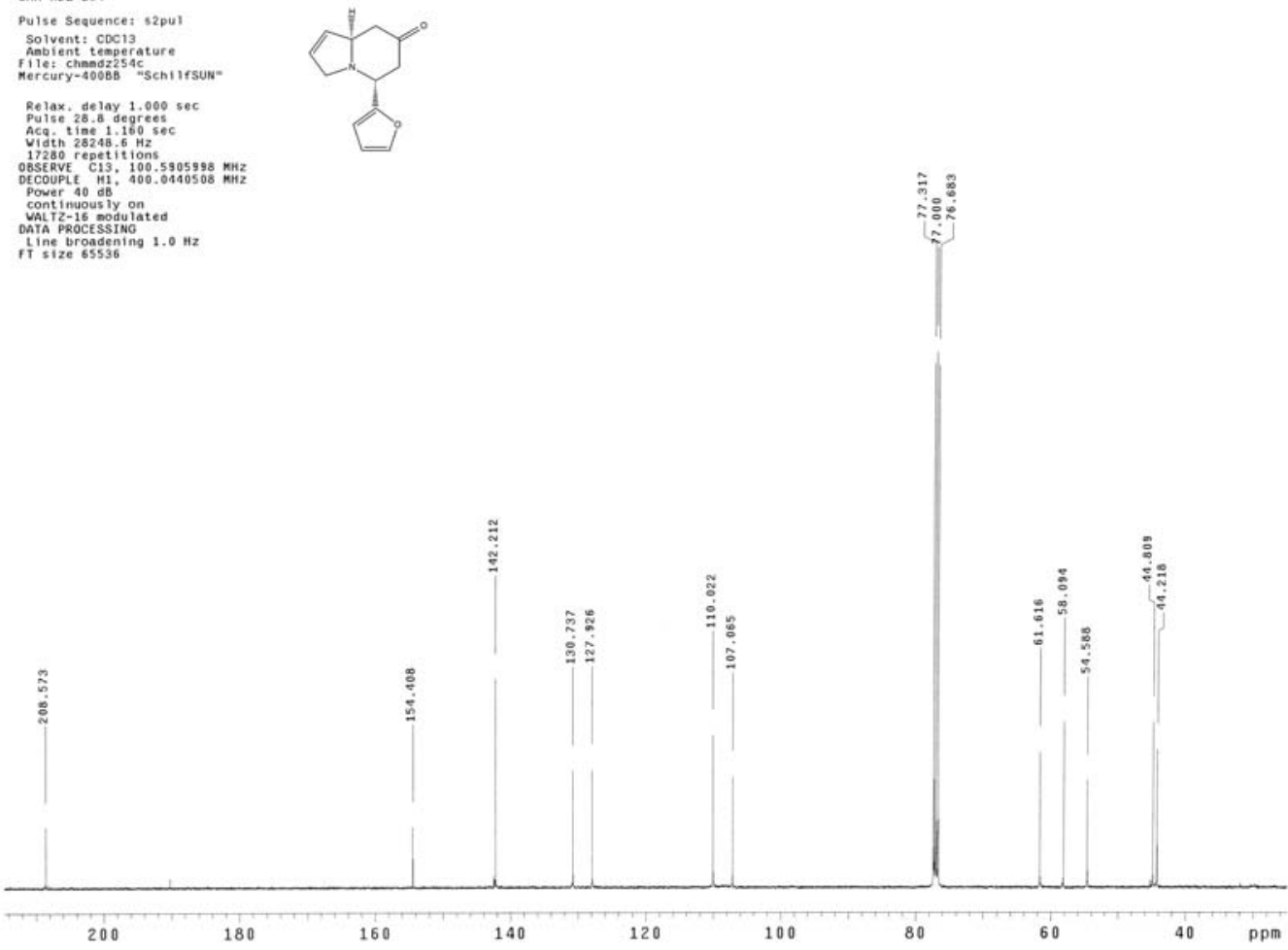

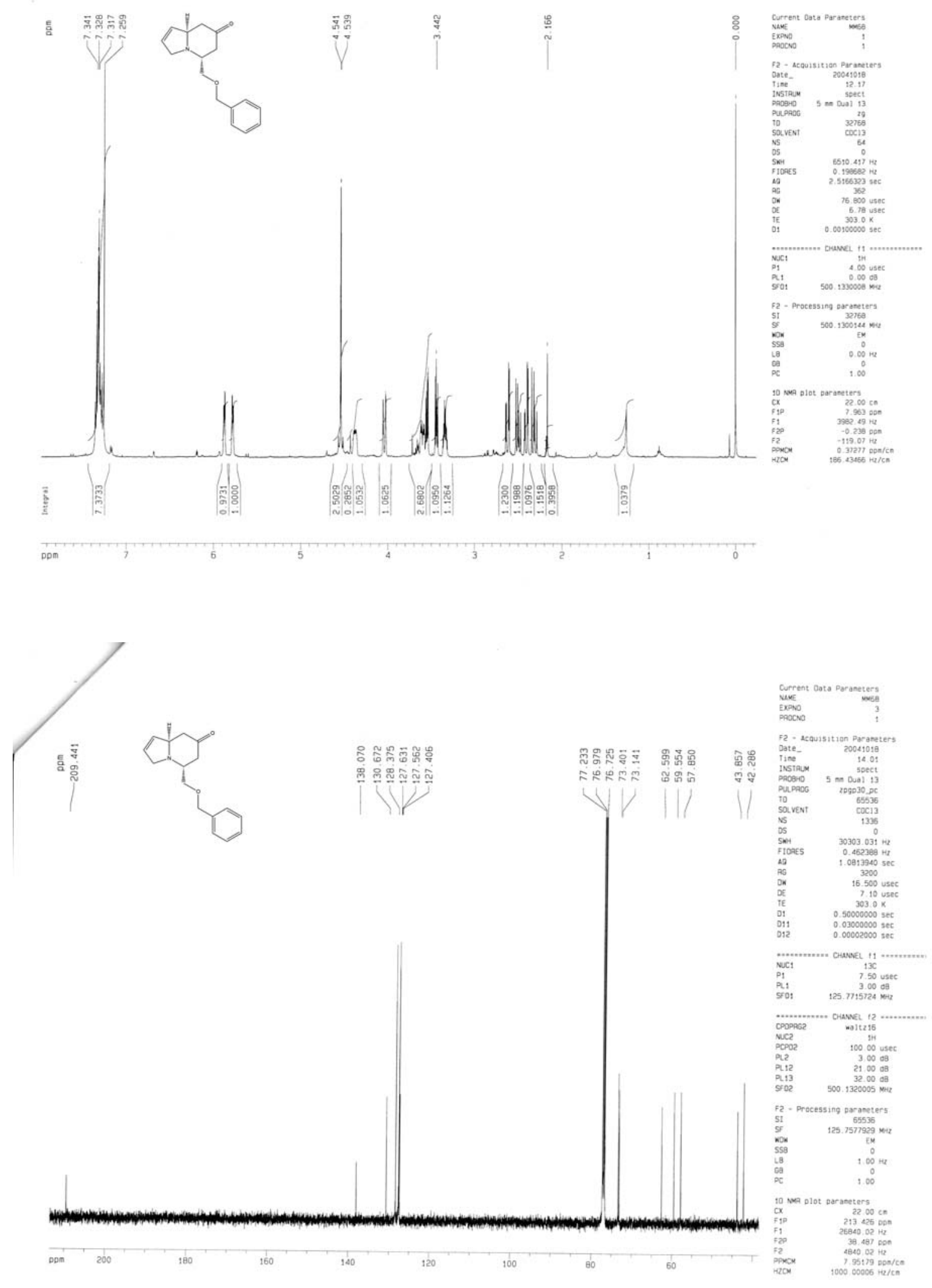


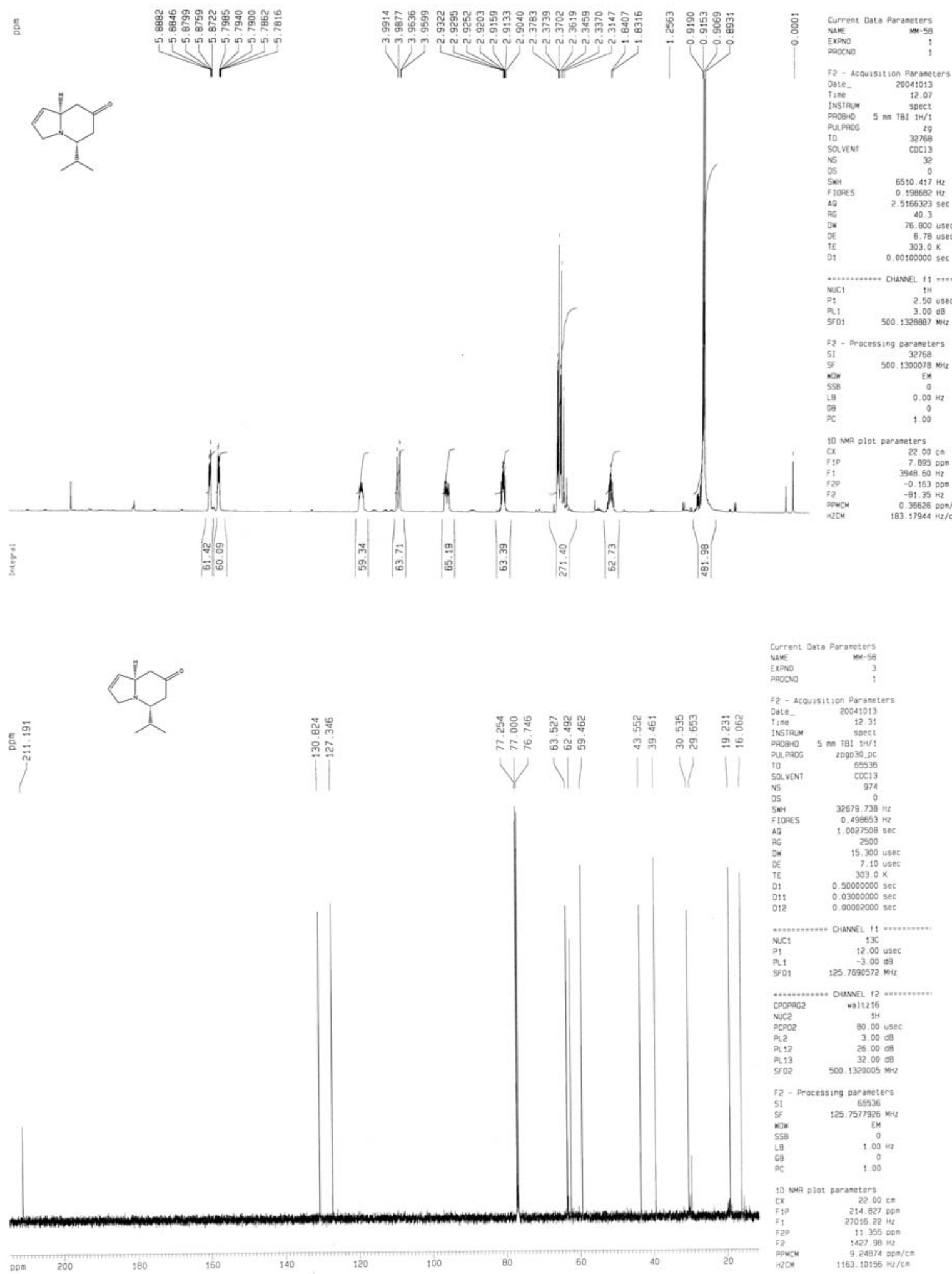




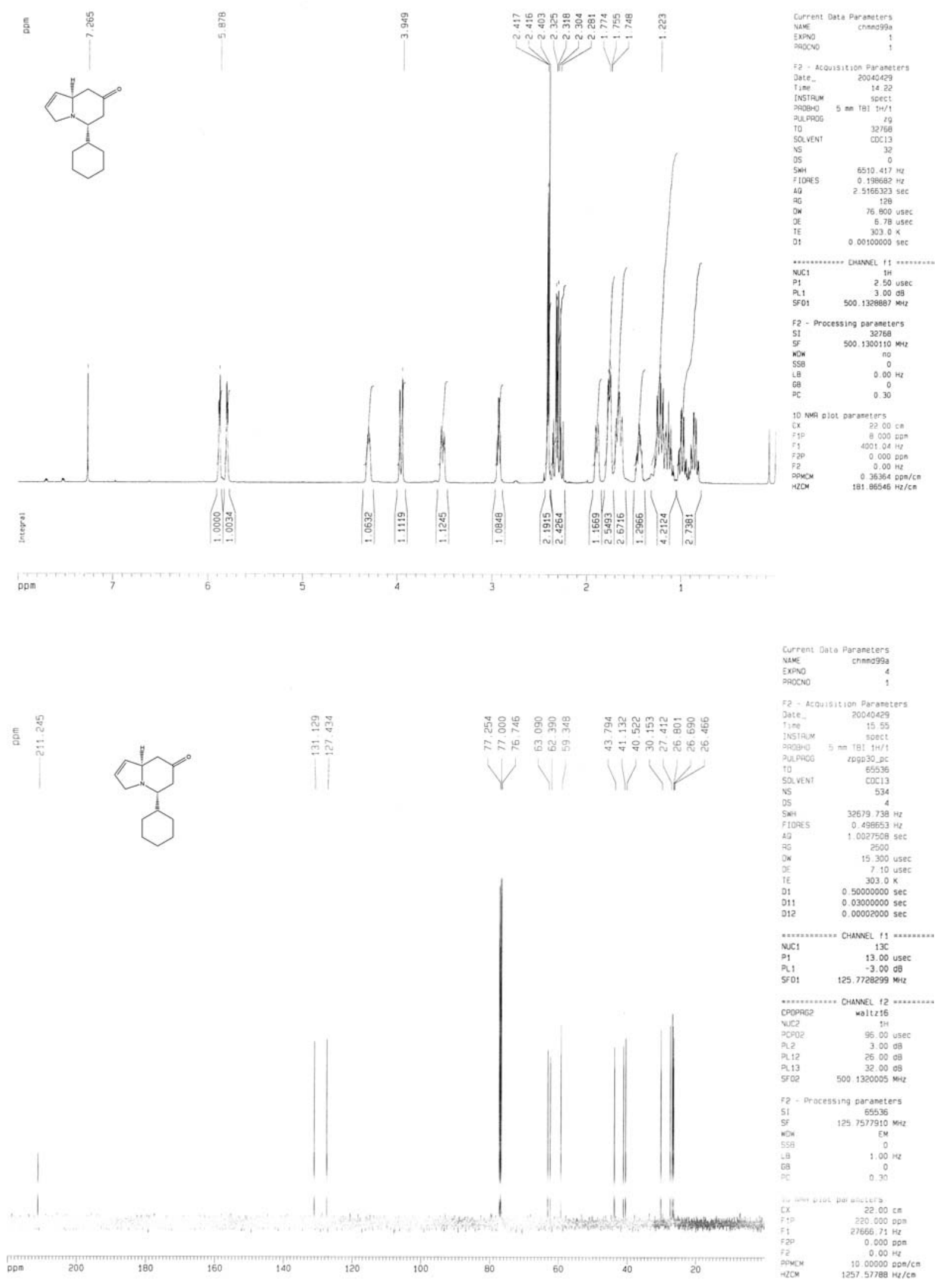



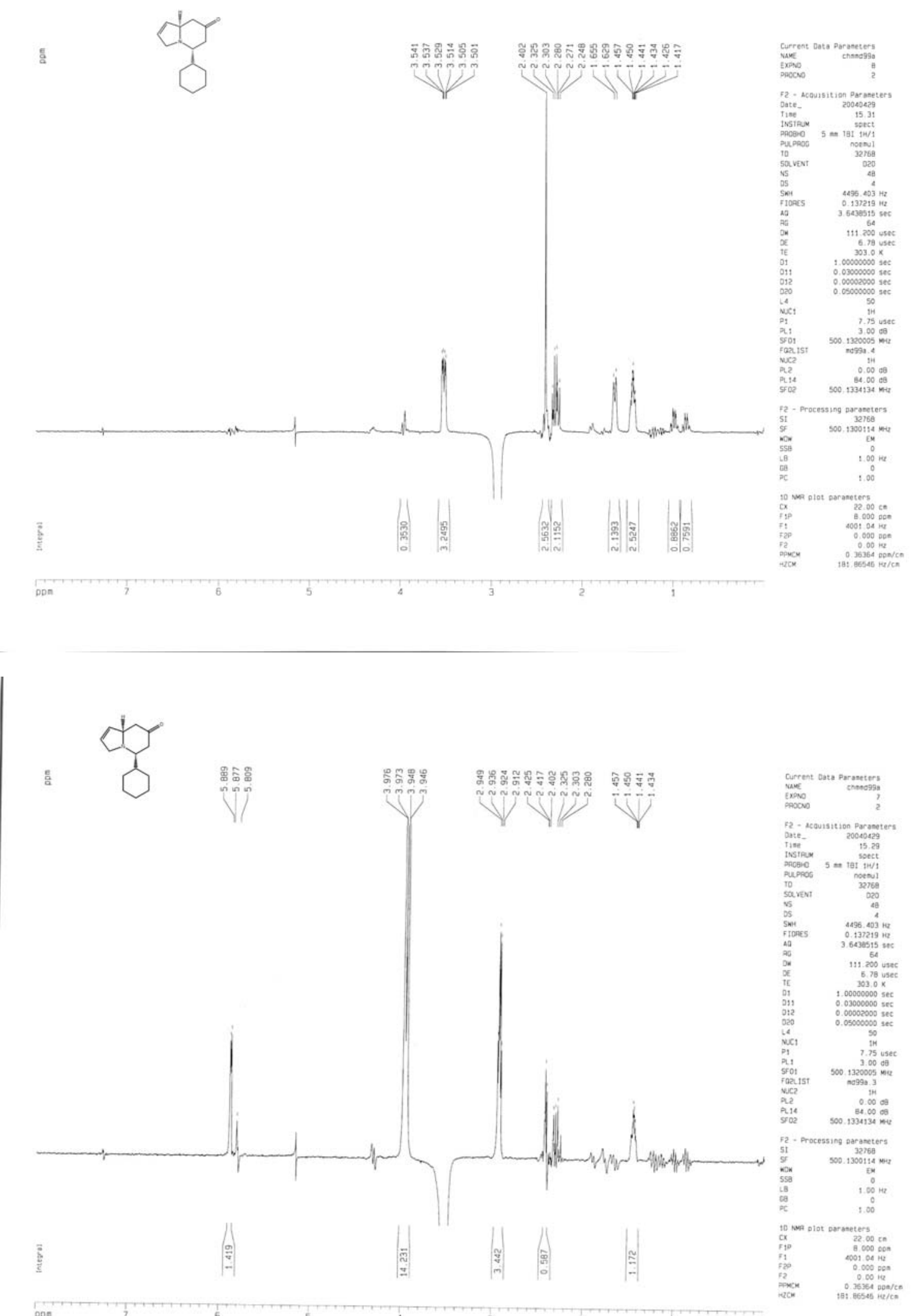

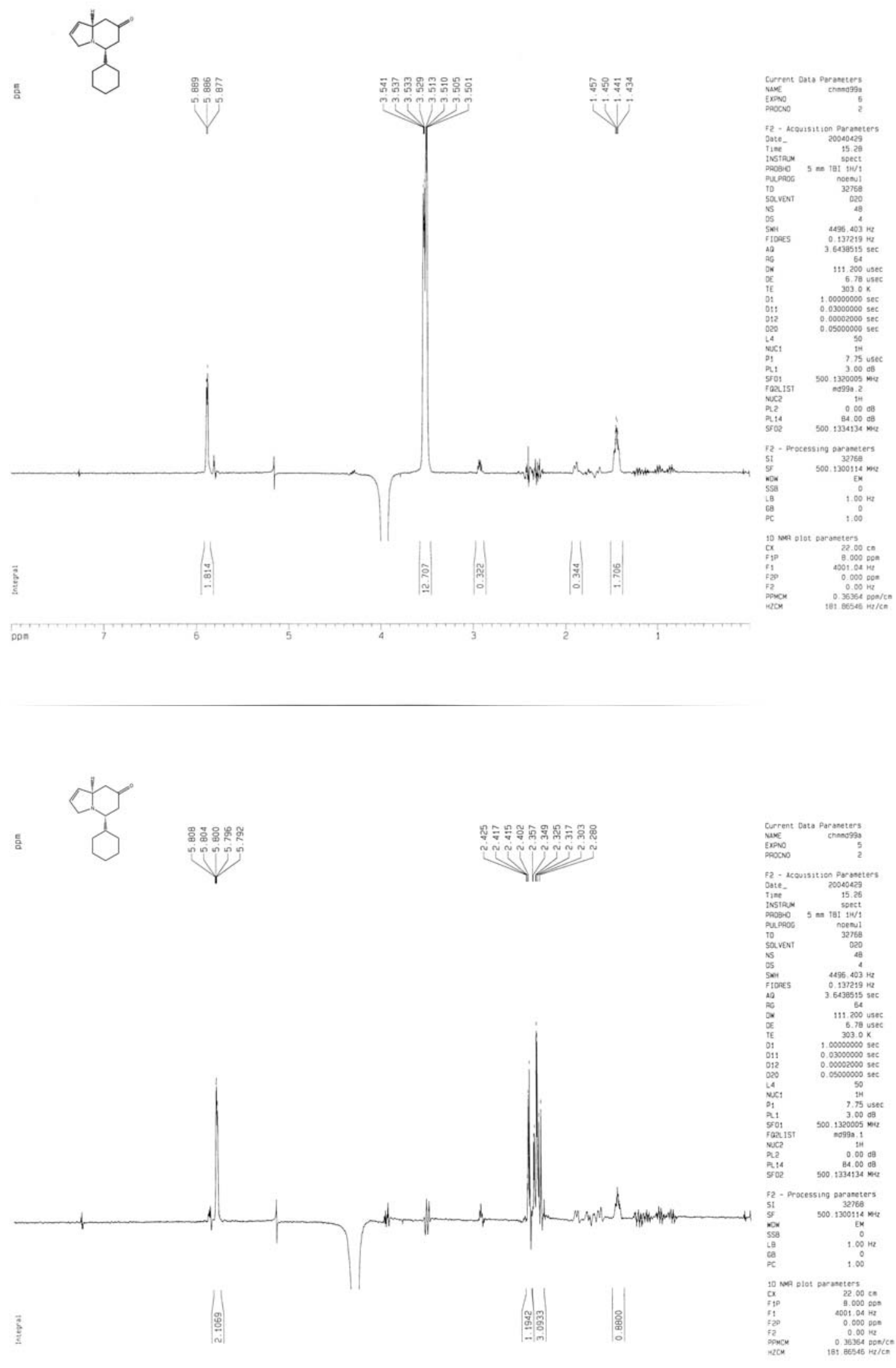

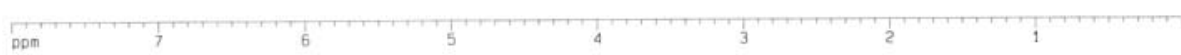

:

虽

:

:

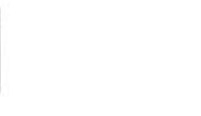

24 

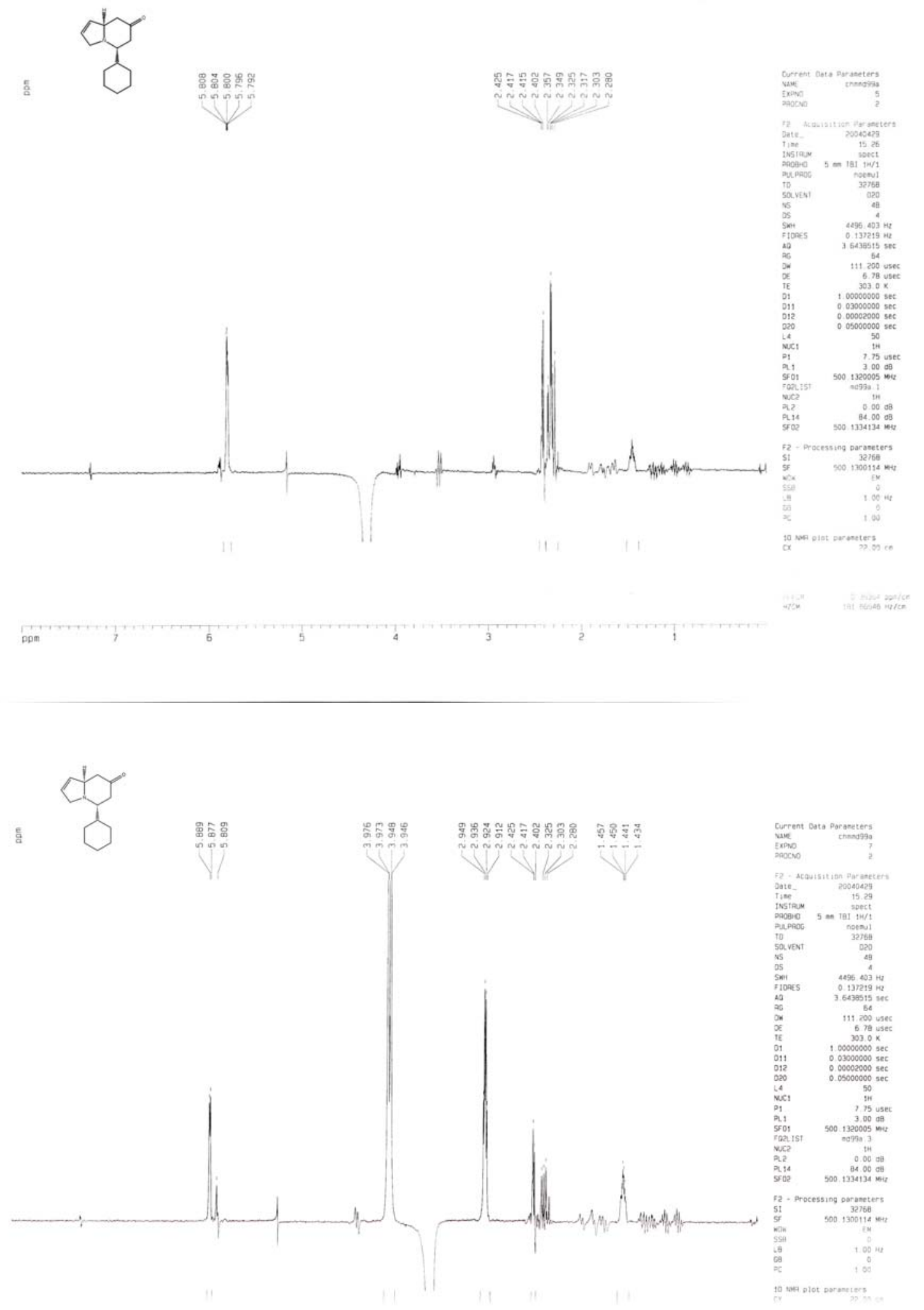


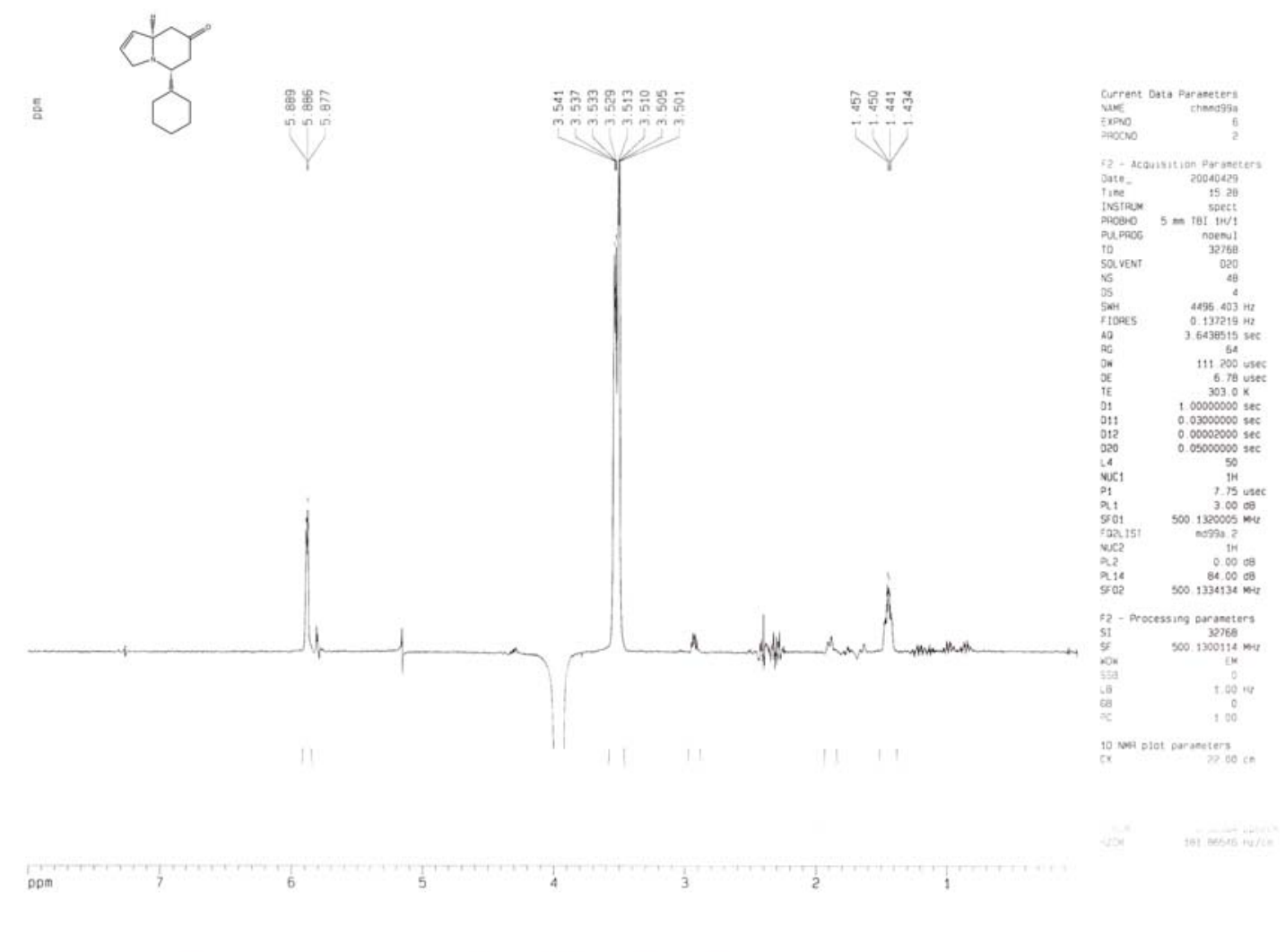

sy
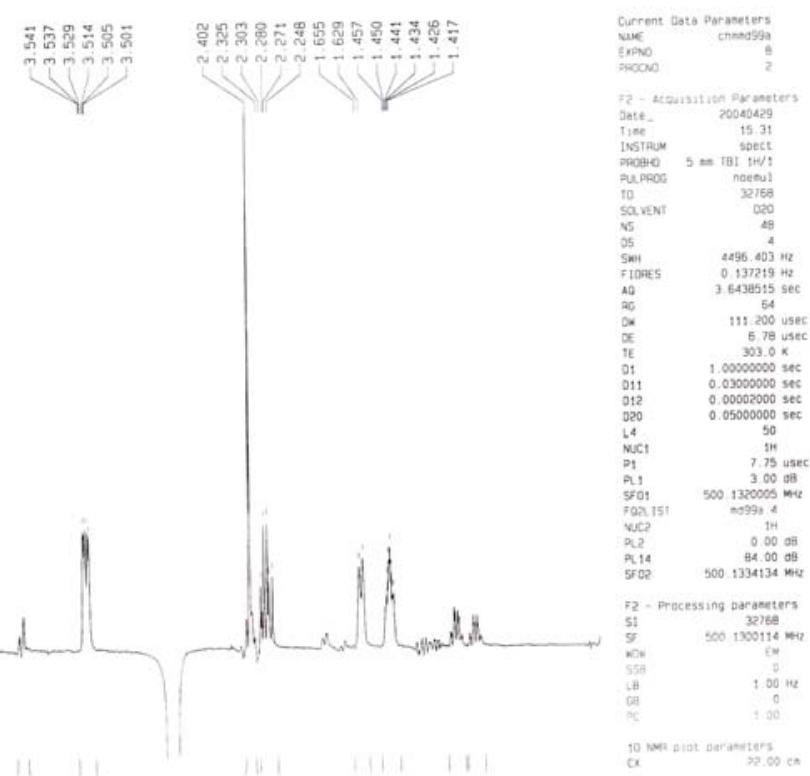

ppon
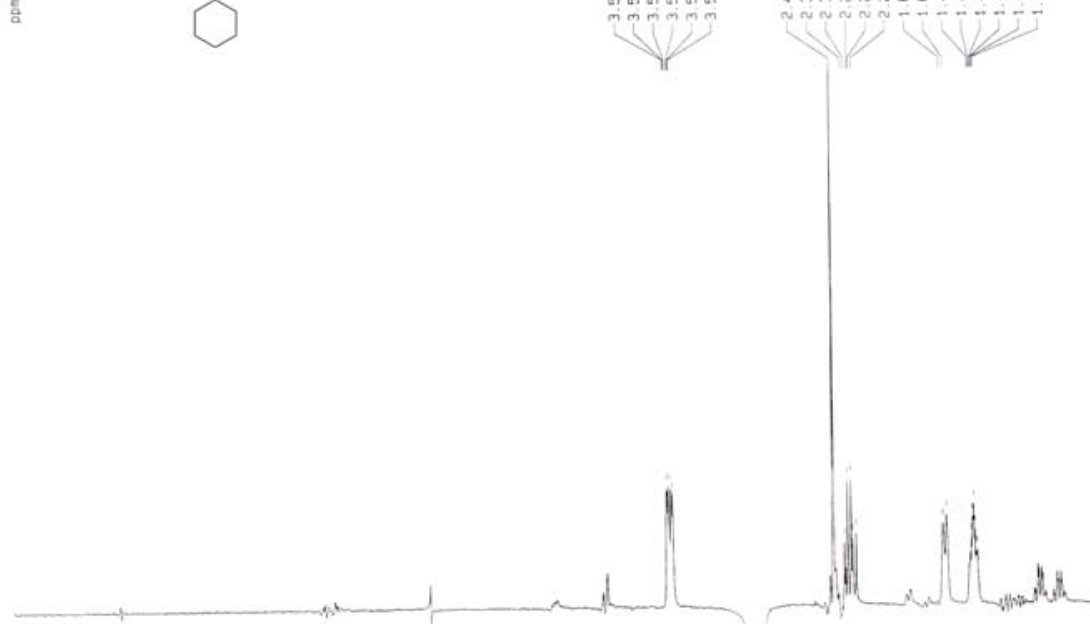

jesin 